\title{
High-Rate Distributed Space-Time-Frequency Coding for Wireless Cooperative Networks
}

\author{
Jinsong Wu, Member, IEEE, Honggang Hu, and Murat Uysal, Senior Member, IEEE
}

\begin{abstract}
In this paper, we propose high-rate distributed space-time-frequency codes (DSTFCs) to exploit maximum achievable diversity gains over frequency-selective fading channels. The proposed designs achieve full-rate for any number of cooperative nodes, and allow channel variations over multiple OFDM blocks within one DSTFC codeword. We analyze diversity gains of DSTFCs through both conditional and average pairwise error probability (PEP), and we proposes better design criteria based on one-side channel conditional PEP. We show that the difference between the frequency-selective channel orders of source-to-relay and relay-to-destination links may provide extra diversity advantages, thus additional performance gains. Through Monte-Carlo simulations, we demonstrate that proposed high-rate DSTFCs provide notable diversity advantages over existing designs.
\end{abstract}

Index Terms-Amplify-and-forward relaying, high-rate, distributed space-time-frequency coding, one-side conditional pairwise error probability, diversity.

\section{INTRODUCTION}

$\mathbf{T}$ HE performance of wireless communications is highly degraded in the presence of fading, shadowing, and multiuser interference. To overcome these limitations, cooperative diversity has been proposed [1], [2] which extracts spatial diversity advantages in a distributed manner by creating a virtual antenna array. Relay-based cooperative communications can notably increase capacity, and this capacity gain can also be translated into reduced power for the users [3].

To extract the spatial diversity in distributed scenarios, a number of distributed space-time codes (DSTCs) have been developed for cooperative communications over frequency-flat channels [2], [4], [5]. On the other hand, broadband channels exhibit frequency-selectivity and require the deployment of transceiver techniques which will handle the resulting intersymbol interference (ISI). Orthogonal frequency division multiplexing $(\mathrm{OFDM})$ is a kind of multicarrier communication system where the high-rate data stream is demultiplexed and transmitted over a number of frequency subcarriers. If the subcarrier width is sufficiently small compared to the channel coherence bandwidth, a frequency-flat channel model can be assumed for each subcarrier and channel distortion can be easily compensated at the receiver.

Manuscript received April 15, 2010; revised August 28, 2010; accepted October 27, 2010. The associate editor coordinating the review of this paper and approving it for publication was B. Sundar Rajan.

J. Wu and $\mathrm{H}$. Hu are with the Department of Electrical and Computer Engineering, University of Waterloo, Waterloo, Ontario, Canada N2L 3G1 (e-mail: wujs@ieee.org, h7hu@uwaterloo.ca).

M. Uysal is with the Faculty of Engineering, Özyeğin University, 34662, Istanbul, Turkey (e-mail: murat.uysal@ozyegin.edu.tr).

$\mathrm{H}$. Hu is supported by the National Natural Science Foundation of China under Grant No. 60802029.

Digital Object Identifier 10.1109/TWC.2011.120810.100615
There has been a growing attention on the design of cooperative OFDM systems [6]-[8]. Seddik and Liu have proposed distributed space-frequency codes (DSFCs) assuming both decode-and-forward (DF) and amplify-and-forward (AF) relaying [7]. The proposed code structure of AF DSFC is only shown to achieve full diversity for some special cases [7]. In [6], Zhang et al. have introduced some AF DSFCs and provided diversity analysis for arbitrary number of relay nodes and channel lengths based on some approximations. The maximal rate of AF-DSFCs proposed in both [7] and [6] is one OFDM block per two-phase cooperative OFDM transmission, which is termed as "full rate". Wang, et al. have proposed two lower-rate (i.e., less than full rate) DSFCs to combat multiple carrier frequency offsets (CFO) [8].

Space-time-frequency codes (STFCs) [9] have been further proposed for use in cooperative OFDM systems with multiple relays over multiple OFDM blocks and multiple subcarriers [10]-[12]. Oguz et al. have proposed distributed STFC (DSTFC) for a two-user multiple-access-channel with cooperating transmitters [10]. The DSTFC design proposed by Tran et al. [11] is to improve the system performance of multi-band OFDM-based ultra-wideband communications using the cooperation of two nodes. Yang et al. have studied space-time-frequency block coding for DF relaying version [12]. All the existing DSTFC designs [10]-[12] are based on orthogonal space-time block-codes [13], which introduce some limitations to applications. The current designs [10][12] only consider two cooperative nodes. In practice, usually more than two cooperative nodes are available. Therefore, extending those designs for more than two cooperative nodes is of practical concern. However, orthogonal designs [14] based approaches cannot achieve full-rate if the number of cooperative nodes is more than two. It should be also noted that orthogonal designs ensure simpler maximum-likelihood receiver; however, this requires that the channels are constant over multiple OFDM blocks, that is to say, this prevents from exploiting time diversity over multiple OFDM blocks.

To overcome the design limitations mentioned above, in this paper, we propose high-rate DSTFC for relay-based AF cooperative communications. The proposed designs achieve full-rate for any number of cooperative nodes, and allow channel variations over multiple OFDM blocks within one DSTFC codeword. Unlike the designs of AF DSFC which were based on diversity analyses using average pairwise error probability (PEP) [6], [7], we design AF DSFC using oneside-channel conditioned PEP based diversity analysis, which enables to shed light on code construction. In addition, we also provide some discussions on average PEP based diversity analysis for our design creteria, and show some insights on 
differences between conditional and average PEP-based based designs.

Notations: $j=\sqrt{-1},(\cdot)^{\mathcal{T}}$ matrix transpose, $(\cdot)^{*}$ conjugate, $(\cdot)^{\mathcal{H}}$ matrix conjugate transpose, $\circ$ Hadamard product operator, $\phi$ denotes empty set, $\otimes$ Kronecker product, $\delta(\cdot)$ Kronecker delta, $[\mathbf{A}]_{a, b}$ the $(a, b)$ entry (element) of matrix $\mathbf{A}, \operatorname{tr}(\cdot)$ matrix trace operation, $\operatorname{Re}(\cdot)$ real part of the object (matrix or variable), Im $(\cdot)$ imaginary part of the object (matrix or variable), $[\mathbf{A}]^{. a}$ element-wise power of $a$ for matrix $\mathbf{A}$, and $\zeta_{a}=\exp \left(j \frac{2 \pi}{a}\right)$, and $\mathrm{E}_{\alpha}(\cdot)$ expectation over random variable or random variable set $\alpha$.

\section{SyStem MOdEL AND PROPOSED APPROACH}

We consider a wireless cooperative communication system which consists of a source node, $K$ relay nodes, and a destination node. The source node sends $N_{C}$ symbols over $N_{C}$ OFDM subcarriers per transmission. Perfect synchronization is assumed for all transmissions between different relay nodes and the destination node. One OFDM block transmission in the half-duplex cooperative-communications consists of two phases. In the first phase, the source node adds cyclic-prefix (CP) and broadcasts the OFDM block to all $K$ relay nodes. Each relay node receives the channel symbols with additive noise at that relay. During the second phase, the source node stops the transmission. All relay nodes remove the $\mathrm{CP}$ and process the received block, i.e., energy normalization, precoding, power-amplifying, etc. Then, all relay nodes $(k=$ $1, \ldots, K)$ simultaneously retransmit the processed signals to the destination node .

The frequency-selective channels are modeled using widely adopted discrete symbol-spaced tap-delay-line (SSTDL) [15]. The channel between the source node and the $k$-th relay node in the $t$-th OFDM block experiences frequency-selective, temporally flat Rayleigh fading with channel coefficients $\mathbf{h}^{(k, t)}=\left[h_{(0)}^{(k, t)}, \ldots, h_{L_{h}^{(k)}}^{(k, t)}\right]^{\mathcal{T}}, k=1, \ldots, K$. Similarly, the channel coefficient vector between the $k$-th relay node and the destination node in the $t$-th OFDM block is represented by $\mathbf{g}^{(k, t)}=\left[g_{(0)}^{(k, t)}, \ldots, g_{L_{g}^{(k)}}^{(k, t)}\right]^{\mathcal{T}}, k=1, \ldots, K$. Set $L=$ $\max \left\{L_{h}^{(k)}, L_{g}^{(k)}, k=1, \ldots, K\right\}$ where $L_{h}^{(k)}$ and $L_{g}^{(k)}$ are the frequency-selective channel orders for source-to-relay and relay-to-destination links, respectively. The entries of $\mathbf{h}^{(k, t)}$ and $\mathbf{g}^{(k, t)}$ are Rayleigh fading channel gains, and are modeled as complex Gaussian with zero mean, whose variances depend on power delay profile. We assume that the channel coefficients remain constant within one OFDM block and vary independently among different OFDM blocks. We further assume channel power constraints as $E\left(\left(\mathbf{h}^{(k, t)}\right)^{\mathcal{H}} \mathbf{h}^{(k, t)}\right)=1$ and $E\left(\left(\mathbf{g}^{(k, t)}\right)^{\mathcal{H}} \mathbf{g}^{(k, t)}\right)=1$. Let $H_{p}^{(k, t)}$ denote the $p$-th subcarrier channel gain from the source node to the $k$-th relay node during the $t$-th OFDM block. It is given by

$$
H_{p}^{(k, t)}=\sum_{l=o}^{L_{h}^{(k)}} h_{(l)}^{(k, t)} e^{-j\left(2 \pi / N_{c}\right) l(p-1)}=\mathbf{w}_{p}^{\left(L_{h}^{(k)}\right)} \mathbf{h}^{(k, t)}
$$

where $\mathbf{w}_{p}^{(L)}=\left[1, \omega^{p-1}, \omega^{2(p-1)}, \cdots, \omega^{L(p-1)}\right]^{\mathcal{T}}$. Let $G_{p}^{(k, t)}$ denote the $p$-th subcarrier channel gain from the $k$-th relay node to the destination node during the $t$-th OFDM block. It is given by

$$
G_{p}^{(k, t)}=\sum_{l=o}^{L_{g}^{(k)}} g_{(l)}^{(k, t)} e^{-j\left(2 \pi / N_{c}\right) l(p-1)}=\mathbf{w}_{p}^{\left(L_{g}^{(k)}\right)} \mathbf{g}^{(k, t)} .
$$

One DSTFC codeword is of size $N_{F} \times K \times T$, i.e., across $K$ relays, $T$ OFDM blocks, and $N_{F}$ subcarriers. Thus, $T$ OFDM blocks contain $n=\frac{N_{C}}{N_{F}}$ DSTFC codewords, and the rest of subcarriers may be padded by zero. The construction and transmission procedure for the $i$-th DSTFC codeword, $i=1, \ldots, n$, is described as follows: At the source node, a data symbol vector of size $N_{F} T \times 1, \mathbf{x}_{i}$, is encoded in source node by

$$
\mathbf{x}_{i}=\theta \mathbf{s}_{i}, i=1, \ldots, n
$$

where $\mathbf{s}_{i}$ is the source data vector carved from the signal alphabet $\mathcal{A} \in \mathbb{Z}[j]$. The matrix $\theta$ is designed such that, for any two different non-zero vector $\mathbf{s}_{i}^{(1)}$ and $\mathbf{s}_{i}^{(2)},\left[\mathbf{x}_{i}^{(1)}-\mathbf{x}_{i}^{(2)}\right]_{a, 1} \neq 0$ always holds for all $a=1, \ldots, N_{F} T$, where $\mathbf{x}_{i}^{(1)}=\theta \mathbf{s}_{i}^{(1)}$ and $\mathbf{x}_{i}^{(2)}=\theta \mathbf{s}_{i}^{(2)}$. The design of $\theta$ can be carried out using signal-space-diversity concepts [16], [17]. For example, $\theta$ can be constructed as $\theta=\left[\mathbf{F}_{N_{s}}\right]^{\mathcal{H}} \operatorname{diag}\left(1, \alpha, \ldots, \alpha^{N_{s}-1}\right)$, where $N_{s}$ should be chosen as a power of $2, N_{s}=N_{F} T, \alpha=$ $\exp \left(j \frac{2 \pi}{4 N_{s}}\right)$, where $\left[\mathbf{F}_{N_{s}}\right]_{a_{1}, a_{2}}=\exp \left(j \frac{2 \pi\left(a_{1}-1\right)\left(a_{2}-1\right)}{N_{s}}\right)$ [16]-[19].

Denote $\mathbf{x}^{(i, t)}=\left[\mathbf{x}_{i}\right]_{(t-1) N_{F}+1: t N_{F}, 1}$. During the $t$-th OFDM block, using subcarriers $\left\{p_{1}^{(i)}, \ldots, p_{N_{F}}^{(i)}\right\}$, the source node transmits the sequence $\mathbf{x}^{(i, t)}$ of size $N_{F} \times 1$ to the relay node. The received signal vector $\mathbf{r}_{f}^{(i, k, t)}$ at the $k$-th relay node in frequency domain is given by

$$
\mathbf{r}_{f}^{(i, k, t)}=\sqrt{P_{1}} \mathbf{x}^{(i, t)} \circ \mathbf{h}_{f}^{(i, k, t)}+\mathbf{v}_{f}^{(i, k, t)},
$$

where $\mathbf{h}_{f}^{(i, k, t)}=\left[H_{p_{1}^{(i)}}^{(k, t)}, \cdots, H_{p_{N_{F}}^{(i)}}^{(k, t)}\right], \mathbf{v}_{f}^{(i, k, t)}$ is the relevant complex Gaussian noise vector at the $k$-th relay node, and $E\left(\mathbf{v}_{f}^{(i, k, t)}\left(\mathbf{v}_{f}^{(i, k, t)}\right)^{\mathcal{H}}\right)=\mathbf{I}_{N_{F}}$.

At the destination node, the received signal vector in frequency domain is

$$
\begin{aligned}
& \mathbf{y}_{f}^{(i, t)}=\mathbf{u}_{f}^{(i, t)} \\
& +\sqrt{P_{1}} \sum_{k=1}^{K}\left(\beta^{(k)} \sqrt{P_{2}^{(k)}} \mathbf{a}^{(i, k, t)} \circ \mathbf{r}_{f}^{(i, k, t)} \circ \mathbf{g}_{f}^{(i, k, t)}\right),
\end{aligned}
$$

where $\mathbf{g}_{f}^{(i, k, t)}=\left[G_{p_{1}^{(i)}}^{(k, t)}, \cdots, G_{p_{N_{F}}^{(i)}}^{(k, t)}\right], \beta^{(k)}=\frac{1}{\sqrt{P_{1}+1}}, \mathbf{u}_{f}^{(i, k, t)}$ is the relevant complex Gaussian noise vector at the destination node, and $E\left(\mathbf{u}_{f}^{(i, t)}\left(\mathbf{u}_{f}^{(i, t)}\right)^{\mathcal{H}}\right)=\mathbf{I}_{N_{F}}$. The design of $\mathbf{a}^{(k, t)}$ will be later discussed in Section III and Appendices A-B.

Combining (4) with (5), the received symbol vector during the $t$-th OFDM block can be rewritten as

$$
\begin{aligned}
& \mathbf{y}_{f}^{(i, t)}=\overline{\mathbf{u}}_{f}^{(i, t)} \\
& +\sqrt{P_{1}} \operatorname{diag}\left(\mathbf{x}^{(i, t)}\right) \sum_{k=1}^{K}\left(\beta^{(k)} \sqrt{P_{2}^{(k)}} \overline{\mathbf{h}}_{f}^{(i, k, t)}\right),
\end{aligned}
$$


where $\overline{\mathbf{h}}_{f}^{(i, k, t)}=\mathbf{a}^{(i, k, t)} \circ \mathbf{h}_{f}^{(i, k, t)} \circ \mathbf{g}_{f}^{(i, k, t)}$, and $\overline{\mathbf{u}}_{f}^{(i, t)}=$ $\mathbf{u}_{f}^{(i, t)}+\sum_{k=1}^{K}\left(\sqrt{P_{2}^{(k)}} \beta^{(k)} \mathbf{a}^{(i, k, t)} \circ \mathbf{v}_{f}^{(i, k, t)} \circ \mathbf{g}_{f}^{(i, k, t)}\right)$. Thus, the received symbol vector for the $i$-th DSTFC codeword is given by

$$
\mathbf{y}_{f}^{(i)}=\sqrt{P_{1}} \operatorname{diag}\left(\mathbf{x}^{(i)}\right) \overline{\mathbf{h}}_{f}^{(i)}+\overline{\mathbf{u}}_{f}^{(i)}
$$

where

$$
\begin{aligned}
& \mathbf{x}^{(i)}=\left[\left[\mathbf{x}^{(i, 1)}\right]^{\mathcal{T}}, \ldots,\left[\mathbf{x}^{(i, T)}\right]^{\mathcal{T}}\right]^{\mathcal{T}}, \\
& \overline{\mathbf{u}}_{f}^{(i)}=\left[\left[\overline{\mathbf{u}}_{f}^{(i, 1)}\right]^{\mathcal{T}}, \ldots,\left[\overline{\mathbf{u}}_{f}^{(i, 1)}\right]^{\mathcal{T}}\right]^{\mathcal{T}}, \\
& \mathbf{y}_{f}^{(i)}=\left[\left[\mathbf{y}_{f}^{(i, 1)}\right]^{\mathcal{T}}, \ldots,\left[\mathbf{y}_{f}^{(i, 1)}\right]^{\mathcal{T}}\right]^{\mathcal{T}},
\end{aligned}
$$

$\overline{\mathbf{h}}_{f}^{(i)}=\left[\left[\overline{\mathbf{h}}^{(i, 1)}\right]^{\mathcal{T}}, \ldots,\left[\overline{\mathbf{h}}^{(i, T)}\right]^{\mathcal{T}}\right]^{\mathcal{T}}$, and $\overline{\mathbf{h}}^{(i, t)}=$ $\sum_{k=1}^{K}\left(\beta^{(k)} \sqrt{P_{2}^{(k)}} \overline{\mathbf{h}}_{f}^{(i, k, t)}\right)$. The received vectors are fed to maximum likelihood (ML) decoder given by

$$
\begin{aligned}
& \underset{\mathbf{s}_{i}}{\arg \max }\left(\mathbf{y}_{f}^{(i)} \mid \mathbf{s}_{i}\right)= \\
& \underset{\mathbf{s}_{i}}{\arg \min }\left\|\mathbf{y}_{f}^{(i)}-\sqrt{P_{1}} \operatorname{diag}\left(\mathbf{x}^{(i)}\right) \overline{\mathbf{h}}_{f}^{(i)}\right\|^{2} .
\end{aligned}
$$

\section{Diversity Analysis ANd Design CRiteria}

In this section, in order to analyze the diversity gains, we first derive conditional PEP of the $i$-th codeword of the DSTFC under consideration and discuss relevant code design parameters. Second, we present an average PEP based analysis and point out the differences between average and conditional PEP based designs.

\section{A. Conditional PEP based analysis and code design}

Given the channel coefficients $\mathbf{h}_{f}^{(i, k, t)}, \mathbf{g}_{f}^{(i, k, t)}$, $k=1, \ldots, K, t=1, \ldots, T$, the conditional PEP can be bounded by

$$
\begin{aligned}
& \operatorname{Pr}\left(\mathbf{x}^{(i)} \rightarrow \tilde{\mathbf{x}}^{(i)} \begin{array}{l}
\begin{array}{l}
\mathbf{h}_{f}^{(i, k, t)}, \mathbf{g}_{f}^{(i, k, t)} \\
k=1, \ldots, K, t=1, \ldots, T
\end{array} \\
\leq \exp \left(-\frac{\left\|\sqrt{P_{1}} \Delta^{(i)} \overline{\mathbf{h}}_{f}^{(i)}\right\|^{2}}{2 E_{\left\{\mathbf{v}_{f}^{(i, k, t)}, \mathbf{u}_{f}^{(i, t)}\right\}}\left(\left\|\overline{\mathbf{u}}_{f}^{(i)}\right\|^{2}\right)}\right),
\end{array}\right.
\end{aligned}
$$

where $\Delta^{(i)}=\operatorname{diag}\left(\mathbf{x}^{(i)}-\widetilde{\mathbf{x}}^{(i)}\right)$. The expectation of $\mathbf{u}_{f}^{(i, t)}$ over $\left\{\mathbf{v}_{f}^{(i, k, t)}, \mathbf{u}_{f}^{(i, t)}\right\}$ can be performed as

$$
\begin{aligned}
& E_{\left\{\mathbf{v}_{f}^{(i, k, t)}, \mathbf{u}_{f}^{(i, t)}\right\}}\left(\left\|\overline{\mathbf{u}}_{f}^{(i)}\right\|^{2}\right)= \\
& \sum_{t=1}^{T}\left(E_{\left\{\mathbf{v}_{f}^{(i, k, t)}, \mathbf{u}_{f}^{(i, t)}\right\}}\left(\left\|\overline{\mathbf{u}}_{f}^{(i, t)}\right\|^{2}\right)\right),
\end{aligned}
$$

where

$$
\begin{gathered}
E_{\left\{\mathbf{v}_{f}^{(i, k, t)}, \mathbf{u}_{f}^{(i, t)}\right\}}\left(\left\|\overline{\mathbf{u}}_{f}^{(i, t)}\right\|^{2}\right) \\
=\operatorname{tr}\left(E_{\left\{\mathbf{u}_{f}^{(i, t)}\right\}}\left(\mathbf{u}_{f}^{(i, t)}\left(\mathbf{u}_{f}^{(i, t)}\right)^{H}\right)\right) \\
+\frac{1}{1+P_{1}} \sum_{k=1}^{K} \operatorname{tr}\left(P_{2}^{(k)} \mathbf{a}_{\mathbf{g}}^{(i, t)} \mathbf{R}_{\mathbf{v}_{f}}^{(i, k, t)}\left(\mathbf{a}_{\mathbf{g}}^{(i, t)}\right)^{H}\right) \\
=N_{F}+\frac{1}{1+P_{1}} \sum_{k=1}^{K}\left(P_{2}^{(k)}\left(\mathbf{g}_{f}^{(i, k, t)}\right)^{H} \mathbf{g}_{f}^{(i, k, t)}\right),
\end{gathered}
$$

Here

$$
\begin{gathered}
\mathbf{R}_{\mathbf{v}_{f}^{(i, k, t)}}^{\left(i, E^{2}\right.} E_{\left\{\mathbf{v}_{f}^{(i, k, t)}\right\}}\left(\mathbf{v}_{f}^{(i, k, t)}\left(\mathbf{v}_{f}^{(i, k, t)}\right)^{H}\right), \\
\mathbf{a}_{\mathbf{g}}^{(i, k, t)}=\operatorname{diag}\left(\mathbf{a}^{(i, k, t)} \circ \mathbf{g}_{f}^{(i, k, t)}\right),
\end{gathered}
$$

and

$$
\operatorname{diag}\left(\mathbf{a}^{(i, k, t)} \circ\left(\mathbf{a}^{(i, k, t)}\right)^{\mathcal{H}}\right)=\mathbf{I}_{N_{F}} .
$$

The equality in (10) is based on the assumption that the equivalent noise vectors, $\overline{\mathbf{u}}_{f}^{(i, t)}, t=1, . ., T$, are independent.

Now we return our attention to the expression $\left\|\sqrt{P_{1}} \Delta^{(i)} \overline{\mathbf{h}}_{f}^{(i)}\right\|^{2}$ in (9). It can be easily shown that

$$
\left\|\sqrt{P_{1}} \Delta^{(i)} \overline{\mathbf{h}}_{f}^{(i)}\right\|^{2}=P_{1} \operatorname{tr}\left(\Delta^{(i)} \mathbf{A}_{h}^{(i)}\left(\Delta^{(i)}\right)^{\mathcal{H}}\right)
$$

where

$$
\begin{gathered}
\mathbf{A}_{h}^{(i)}=\mathbf{A}^{(i)}\left(\mathbf{h}_{f}^{(i)} \circ \mathbf{g}_{f}^{(i)}\right)\left(\mathbf{h}_{f}^{(i)} \circ \mathbf{g}_{f}^{(i)}\right)^{\mathcal{H}}\left(\mathbf{A}^{(i)}\right)^{\mathcal{H}}, \\
\mathbf{h}_{f}^{(i)}=\left[\left[\mathbf{h}_{f}^{(i, 1)}\right]^{\mathcal{T}}, \ldots,\left[\mathbf{h}_{f}^{(i, T)}\right]^{\mathcal{T}}\right]^{\mathcal{T}}, \\
\mathbf{g}_{f}^{(i)}=\left[\left[\mathbf{g}_{f}^{(i, 1)}\right]^{\mathcal{T}}, \ldots,\left[\mathbf{g}_{f}^{(i, T)}\right]^{\mathcal{T}}\right]^{\mathcal{T}}, \\
\mathbf{A}^{(i)}=\operatorname{diag}\left(\mathbf{A}^{(i, 1)}, \ldots, \mathbf{A}^{(i, T)}\right) \\
\mathbf{h}_{f}^{(i, t)}=\left[\left[\mathbf{h}_{f}^{(i, 1, t)}\right]^{\mathcal{T}}, \ldots,\left[\mathbf{h}_{f}^{(i, K, t)}\right]^{\mathcal{T}}\right]^{\mathcal{T}}, \\
\mathbf{g}_{f}^{(i, t)}=\left[\left[\mathbf{g}_{f}^{(i, 1, t)}\right]^{\mathcal{T}}, \ldots,\left[\mathbf{g}_{f}^{(i, K, t)}\right]^{\mathcal{T}}\right]^{\mathcal{T}}, \\
\mathbf{A}^{(i, t)}=\left[\beta^{(1)} \sqrt{P_{2}^{(1)}} \mathbf{A}^{(i, 1,1)}, \ldots, \beta^{(K)} \sqrt{P_{2}^{(K)}} \mathbf{A}^{(i, K, t)}\right],
\end{gathered}
$$

and $\mathbf{A}^{(i, k, t)}=\operatorname{diag}\left(\mathbf{a}^{(i, k, t)}\right)$.

Using the results in Appendix A, (12) can be rewritten as

$$
\begin{aligned}
& \left\|\sqrt{P_{1}} \Delta^{(i)} \overline{\mathbf{h}}_{f}^{(i)}\right\|^{2}= \\
& P_{1} \operatorname{tr}\left(\Delta^{(i)} \mathrm{M}^{(i)} \psi_{\mathbf{g}} \mathbf{h}(\mathbf{h})^{H}\left(\psi_{\mathbf{g}}\right)^{H}\left(\mathrm{M}^{(i)}\right)^{H}\left(\Delta^{(i)}\right)^{H}\right) \\
& =P_{1}(\mathbf{h})^{H} \mathrm{M}_{\Delta}^{(i)} \mathbf{h},
\end{aligned}
$$

where $\mathrm{M}_{\Delta}^{(i)}=\left(\psi_{\mathbf{g}}\right)^{\mathcal{H}}\left(\mathrm{M}^{(i)}\right)^{\mathcal{H}}\left(\Delta^{(i)}\right)^{\mathcal{H}} \Delta^{(i)} \mathrm{M}^{(i)} \psi_{\mathbf{g}}$. Through averaging over $\left\{\mathbf{h}_{f}^{(i, k, t)}, k=1, \ldots, K, t=1, \ldots, T\right\}$, the PEP conditioned on channel $\left\{\mathbf{g}_{f}^{(i, k, t)}, k=1, \ldots, K, t=1, \ldots, T\right\}$ can be bounded by (14), where the probability density function $\mathcal{P}(\mathbf{h})$ of $\mathbf{h}$ is defined in (15). Denote $\mathbf{R}_{\Delta}^{(i)}$ in (16). Note that 


$$
\begin{aligned}
& \operatorname{Pr}\left(\mathbf{x}^{(i)} \rightarrow \widetilde{\mathbf{x}}^{(i)} \mid\left\{\mathbf{g}_{f}^{(i, k, t)}\right\}, k=1, \ldots, K, t=1, \ldots, T\right) \\
& \leqslant E_{\{\mathbf{h}\}} \exp \left(-\frac{P_{1}(\mathbf{h})^{\mathcal{H}} \mathrm{M}_{\Delta}^{(i)} \mathbf{h}}{2\left(\sum_{t=1}^{T}\left(N_{F}+\frac{1}{1+P_{1}} \sum_{k=1}^{K}\left(P_{2}^{(k)}\left(\mathbf{g}_{f}^{(i, k, t)}\right)^{\mathcal{H}} \mathbf{g}_{f}^{(i, k, t)}\right)\right)\right)}\right)
\end{aligned}
$$

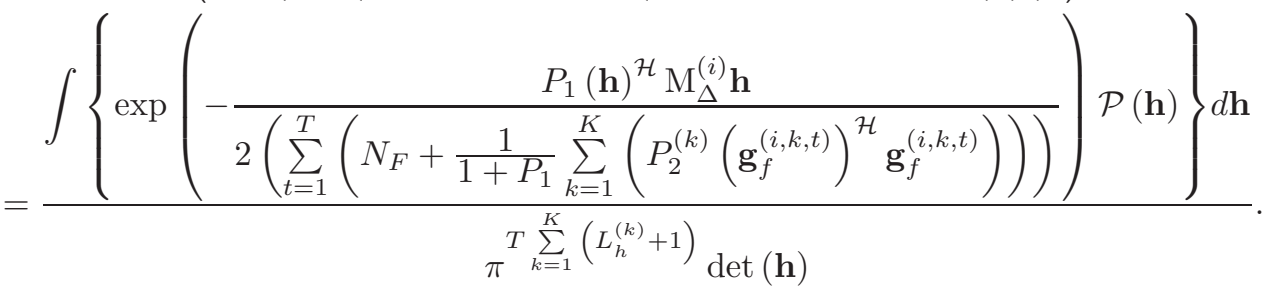

$$
\begin{aligned}
& \mathcal{P}(\mathbf{h})=\frac{1}{\pi^{T \sum_{k=1}^{K}\left(L_{h}^{(k)}+1\right)} \operatorname{det}\left(\mathbf{R}_{\mathbf{h}}\right)} \exp \left\{-(\mathbf{h})^{\mathcal{H}}\left(\mathbf{R}_{\mathbf{h}}\right)^{-1} \mathbf{h}\right\} . \\
& \mathbf{R}_{\Delta}^{(i)}=\left(\frac{P_{1} \mathrm{M}_{\Delta}^{(i)}}{2\left(\sum_{t=1}^{T}\left(N_{F}+\frac{1}{1+P_{1}} \sum_{k=1}^{K}\left(P_{2}^{(k)}\left(\mathbf{g}_{f}^{(i, k, t)}\right)^{\mathcal{H}} \mathbf{g}_{f}^{(i, k, t)}\right)\right)\right)}+\left(\mathbf{R}_{\mathbf{h}}\right)^{-1}\right)^{-1} \\
& \frac{1}{\pi^{T} \sum_{k=1}^{K}\left(L_{h}^{(k)}+1\right) \operatorname{det}\left(\mathbf{R}_{\Delta}^{(i)}\right)} \int\left\{\exp \left(-(\mathbf{h})^{\mathcal{H}}\left(\mathbf{R}_{\Delta}^{(i)}\right)^{-1} \mathbf{h}\right)\right\} d \mathbf{h}=1 . \\
& \operatorname{Pr}\left(\mathbf{x}^{(i)} \rightarrow \widetilde{\mathbf{x}}^{(i)} \mid\left\{\mathbf{g}_{f}^{(i, k, t)}\right\}, k=1, \ldots, K, t=1, \ldots, T\right) \\
& \leq \frac{\operatorname{det}\left(\mathbf{R}_{\Delta}^{(i)}\right)}{\operatorname{det}\left(\mathbf{R}_{\mathbf{h}}\right)}=\frac{1}{\operatorname{det}\left(\left(\mathbf{R}_{\Delta}^{(i)}\right)^{-1} \mathbf{R}_{\mathbf{h}}\right)} \\
& \left.=\frac{1}{\operatorname{det}\left(\frac{P_{1} \mathrm{M}_{\Delta}^{(i)} \mathbf{R}_{\mathbf{h}}}{2\left(\sum_{t=1}^{T}\left(N_{F}+\frac{1}{1+P_{1}} \sum_{k=1}^{K}\left(P_{2}^{(k)}\left(\mathbf{g}_{f}^{(i, k, t)}\right)^{\mathcal{H}} \mathbf{g}_{f}^{(i, k, t)}\right)\right)\right)}+\mathbf{I}_{T} \sum_{k=1}^{K}\left(L_{h}^{(k)}+1\right)\right.}\right) .
\end{aligned}
$$

the relation (17) always holds, and thus (14) can be simplified as (18), where the second equality in (18) is obtained through inserting (16) within.

The corresponding rank and product criteria based on conditional PEP bound in (18) can be expressed as

1) Rank criterion: The minimum rank of $\mathrm{M}_{\Delta}^{(i)} \mathbf{R}_{\mathbf{h}}$ over all pairs of different $\mathbf{x}^{(i)}$ and $\widetilde{\mathbf{x}}^{(i)}$ should be as large as possible.

2) Product criterion: The minimum value of the product of all non-zero eigenvalues of $\mathrm{M}_{\Delta}^{(i)} \mathbf{R}_{\mathbf{h}}$ over all pairs of different $\mathbf{x}^{(i)}$ and $\widetilde{\mathbf{x}}^{(i)}$ should be maximized.

To investigate the maximal achievable conditional diversity order, i.e. the rank of $\mathrm{M}_{\Delta}^{(i)} \mathbf{R}_{\mathbf{h}}$, we further impose the following assumptions:

Assumption 1: Channels for DSTFC are assumed uncorrelated over different relays and OFDM blocks, although the multi-path channels to or from each relay may be correlated, and $\mathbf{R}_{\mathbf{h}}$ is assumed to be full rank, i.e, $\operatorname{rank}\left(\mathbf{R}_{\mathbf{h}}\right)=$ $T \sum_{k=1}^{K}\left(L_{h}^{(k)}+1\right)$.

Assumption 2: Assume that, for all $1 \leq k \leq K$ and $1 \leq$ $t \leq T$, there exists at least one entry in $\mathbf{g}^{(k, t)},\left[\mathbf{g}^{(k, t)}\right]_{c^{(k, t)}, 1}$, such that $\left[\mathbf{g}^{(k, t)}\right]_{c^{(k, t)}, 1} \neq 0,0 \leq c^{(k, t)} \leq L_{g}^{(k)}$, for each $(k, t)$ pair.

The achievable conditional diversity order for conditional PEP in (18) is analyzed in Appendix B along with the design 
of $\mathbf{a}^{(i, k, t)}$, where $k=1, \ldots, K$ and $t=1, \ldots, T$. Those results are summarized in the following theorem.

Theorem 1: Based on discrete frequency-selective channel model for the OFDM-based relay network as described in Section II and under Assumptions 1 and 2, the achievable conditional diversity order of the $i$-th codeword of the proposed DSTFC for PEP conditioned on channel $\left\{\mathbf{g}_{f}^{(i, k, t)}, k=1, \ldots, K, t=1, \ldots, T\right\}$ in (18) is $T \sum_{k=1}^{K}\left(L_{h}^{(k)}+1\right)$. This can be achieved through using

$$
\mathbf{a}^{(i, k, t)}=\left[\mathbf{f}^{(i)}\right]^{\cdot\left((k-1) L_{M}\right)}
$$

and

$$
N_{F} \geq \sum_{k=1}^{K}\left(L_{g}^{(k)}+L_{h}^{(k)}+1\right)
$$

where $L_{M}>\max \left\{\begin{array}{l}\left(L_{g}^{(k)}+L_{h}^{(k)}+1\right), \\ 1 \leq k \leq K\end{array}\right\}, k=1, \ldots, K$, and $t=1, \ldots, T$. These results hold for all $L_{g}^{(k)}<L_{h}^{(k)}$, $L_{h}^{(k)}=L_{g}^{(k)}$, and $L_{g}^{(k)}>L_{h}^{(k)}$ cases.

Example: Based on Theorem 1, one DSTFC design example is provided here. Suppose $N_{C}=32, L_{h}^{(k)}=L_{g}^{(k)}=1$, $k=1,2$, and $K=T=2$, thus $N_{F} \geq 6$. As mentioned earlier in Section II, $N_{F}$ has to be a power of 2 , thus $N_{F}=8, \alpha=\exp \left(j \frac{\pi}{32}\right)$. We therefore have $\mathbf{a}^{(i, k, t)}=$ $\left[\omega^{128(i-1)(k-1)}, \ldots, \omega^{16(8 i-1)(k-1)}\right]^{\mathcal{T}}$, where $i=1,2, k=$ 1,2 , and $t=1,2$.

Now we return our attention to find PEP conditioned on channel $\left\{\mathbf{h}_{f}^{(i, k, t)}, k=1, \ldots, K, t=1, \ldots, T\right\}$. Through averaging (9) over $\left\{\mathbf{g}_{f}^{(i, k, t)}, k=1, \ldots, K, t=1, \ldots, T\right\}$, the PEP conditioned on channel $\left\{\mathbf{h}_{f}^{(i, k, t)}, k=1, \ldots, K, t=1, \ldots, T\right\}$ can be bounded by (21). Using the approximations (22) and (23), (21) can be approximately written as (24). Based on (24), similar to our previous discussions, it can be shown that the achievable conditional diversity order of the $i$-th codeword of the proposed DSTFC is $T \sum_{k=1}^{K}\left(L_{g}^{(k)}+1\right)$. However, unlike the result conditioned on channel $\left\{\mathbf{h}_{f}^{(i, k, t)}, k=1, \ldots, K, t=1, \ldots, T\right\}$, this conditional diversity order bound conditioned on channel $\left\{\mathbf{g}_{f}^{(i, k, t)}, k=1, \ldots, K, t=1, \ldots, T\right\}$ for (24) may be notably larger than the actual achievable conditional diversity order bound due to the use of the aforementioned approximations.

\section{B. Discussions on differences between average and condi- tional PEP -based designs}

In Theorem 1 of [6], it is claimed that, in the case of $L_{h}^{(1)}=$ $\ldots=L_{h}^{(K)}=L_{h}$ and $L_{g}^{(1)}=\ldots=L_{g}^{(K)}=L_{g}$, the achievable diversity for DSFCs is $K \min \left\{L_{h}, L_{g}\right\}$, which implies that, when $L_{h} \neq L_{g}, K\left(\max \left\{L_{h}, L_{g}\right\}-\min \left\{L_{h}, L_{g}\right\}\right)$ extra fading paths cannot provide diversity benefits for system performance. However, according to our present diversity analysis based on conditional PEP, even in the case of $L=$ $L_{h}^{(1)}=\ldots=L_{h}^{(K)}=L_{h}>L_{g} \geq\left\{L_{g}^{(1)}, \ldots, L_{g}^{(K)}\right\}$, the achievable diversity order of DSFC based on conditional PEP can be $K\left(L_{h}+1\right)$. Unlike our design approach, the DSFCs were designed under average PEP based diversity analysis, the resulting $N_{F}$ was set as $K \min \left(L_{h}+1, L_{g}+1\right)$ to achieve their claimed diversity $K \min \left\{L_{h}, L_{g}\right\}$, which actually may not fully exploit available diversity in the relay frequencyselective channels.

To explain the reasons for these somehow conflicting conclusions, we provide the following remarks:

1) The full frequency diversity properties of DSTFC cannot be determined only by the numbers of paths

$$
2 \sum_{k=1}^{K}\left(\min \left(L_{h}^{(k)}+1, L_{g}^{(k)}+1\right)\right),
$$

where the number 2 is used for counting both sides of multipath channels. The extra number of paths

$$
\sum_{k=1}^{K}\left(\begin{array}{l}
\max \left(L_{h}^{(k)}+1, L_{g}^{(k)}+1\right)- \\
\min \left(L_{h}^{(k)}+1, L_{g}^{(k)}+1\right)
\end{array}\right)
$$

may contribute to the system diversity of DSTFC.

2) The diversity analyses using one-side-channel conditional PEP leads to different DSTFC design parameters from those using average PEP. This difference introduces performance gains, which will be verified through simulations in Section IV.

3) There are two kinds of different one-side-channel conditional PEP ( source-to-relay and relay destination ). However, both one-side-channel conditional PEP based diversity analyses will lead to the same design parameters of DSTFC.

4) Note that, in [6], it was claimed that the diversity order be limited by $K \min \left(L_{h}+1, L_{g}+1\right)$. However, we have found that the diversity limitation of $K \min \left(L_{h}+1, L_{g}+1\right)$ is only valid in frequency flat fading relay channels. In the case of $\left\{L_{g}^{(k)}=L_{h}^{(k)}=0, k=1, \ldots k\right\}$, i.e. in flat (nonfrequency-selective) fading channels, the numbers of paths

$$
2 \sum_{k=1}^{K}\left(\min \left(L_{h}^{(k)}+1, L_{g}^{(k)}+1\right)\right)
$$

does fully determine conditional diversity properties of DSTFC for each OFDM block, which is coincidental with average PEP based diversity analysis of DSTC in [20].

To provide some further insights on differences between average and conditional PEP based designs, it is beneficial to also investigate diversity gains directly through deriving average PEP. Here, as an example, we only consider the case of $L_{h}^{(k)}>L_{g}^{(k)}>0, k=1, . ., K$.

Denote $\mathbf{g}=\left[\left[\mathbf{g}^{(1)}\right]^{\mathcal{T}}, \ldots,\left[\mathbf{g}^{(T)}\right]^{\mathcal{T}}\right]^{\mathcal{T}}$ with $\mathbf{g}^{(t)}=$ $\left[\left[\mathbf{g}^{(1, t)}\right]^{\mathcal{T}}, \ldots,\left[\mathbf{g}^{(K, t)}\right]^{\mathcal{T}}\right]^{\mathcal{T}}$. We use set partitioning to categorize whether or not there exists at least one non-zero entry in $\mathbf{g}^{(k, t)}$. Adopting similar proof steps of Appendices A and $\mathrm{B}$ and using $\left\{\mathbf{a}^{(i, k, t)}\right\}$ specified in Theorem 1, the following relations can be proved: 


$$
\begin{aligned}
& \operatorname{Pr}\left(\mathbf{x}^{(i)} \rightarrow \widetilde{\mathbf{x}}^{(i)} \mid\left\{\mathbf{h}_{f}^{(i, k, t)}\right\}, k=1, \ldots, K, t=1, \ldots, T\right) \\
& \leq E_{\left\{\mathbf{g}_{f}^{(i, k, t)}\right\}} \exp \left(-\frac{\left\|\sqrt{P_{1}} \operatorname{diag}\left(\Delta^{(i)}\right) \sum_{k=1}^{K}\left(\beta^{(k)} \sqrt{P_{2}^{(k)}} \overline{\mathbf{h}}_{f}^{(i, k)}\right)\right\|^{2}}{2 E_{\left\{\mathbf{v}_{f}^{(i, k, t)}, \mathbf{u}_{f}^{(i, t)}\right\}}\left(\left\|\overline{\mathbf{u}}_{f}^{(i)}\right\|^{2}\right)}\right) \text {. } \\
& E_{\left\{\mathbf{g}_{f}^{(i, k, t)}\right\}} \exp \left(-\frac{\left\|\sqrt{P_{1}} \operatorname{diag}\left(\Delta^{(i)}\right) \sum_{k=1}^{K}\left(\beta^{(k)} \sqrt{P_{2}^{(k)}} \overline{\mathbf{h}}_{f}^{(i, k)}\right)\right\|^{2}}{2 E_{\left\{\mathbf{v}_{f}^{(i, k, t)}, \mathbf{u}_{f}^{(i, t)}\right\}}\left(\left\|\overline{\mathbf{u}}_{f}^{(i)}\right\|^{2}\right)}\right) \\
& \approx E_{\left\{\mathbf{g}_{f}^{(i, k, t)}\right\}} \exp \left(-\frac{\left\|\sqrt{P_{1}} \operatorname{diag}\left(\Delta^{(i)}\right) \sum_{k=1}^{K}\left(\beta^{(k)} \sqrt{P_{2}^{(k)}} \overline{\mathbf{h}}_{f}^{(i, k)}\right)\right\|^{2}}{2 E_{\left\{\mathbf{v}_{f}^{(i, k, t)}, \mathbf{u}_{f}^{(i, t)}, \mathbf{g}_{f}^{(i, k, t)}\right\}}\left(\left\|\overline{\mathbf{u}}_{f}^{(i)}\right\|^{2}\right)}\right) \\
& E_{\left\{\mathbf{v}_{f}^{(i, k, t)}, \mathbf{u}_{f}^{(i, t)}\right\}}\left(\sum_{t=1}^{T}\left(\sum_{k=1}^{K}\left(P_{2}^{(k)}\left(\mathbf{g}_{f}^{(i, k, t)}\right)^{\mathcal{H}} \mathbf{g}_{f}^{(i, k, t)}\right)\right)\right) \\
& \approx \sum_{t=1}^{T}\left(\sum_{k=1}^{K}\left(P_{2}^{(k)} N_{F}\right)\right)^{k} \text {, } \\
& \operatorname{Pr}\left(\mathbf{x}^{(i)} \rightarrow \widetilde{\mathbf{x}}^{(i)} \mid\left\{\mathbf{h}_{f}^{(i, k, t)}\right\}, k=1, \ldots, K, t=1, \ldots, T\right) \\
& \lesssim E_{\left\{\mathbf{g}_{f}^{(i, k, t)}\right\}} \exp \left(-\frac{\left\|\sqrt{P_{1}} \operatorname{diag}\left(\Delta^{(i)}\right) \sum_{k=1}^{K}\left(\beta^{(k)} \sqrt{P_{2}^{(k)}} \overline{\mathbf{h}}_{f}^{(i, k)}\right)\right\|^{2}}{2 \sum_{t=1}^{T}\left(\sum_{k=1}^{K}\left(P_{2}^{(k)} N_{F}\right)\right)}\right) .
\end{aligned}
$$

1) For $N_{F} \geq \sum_{k=1}^{K}\left(L_{g}^{(k)}+L_{h}^{(k)}+1\right)$, we have

$$
\begin{aligned}
& N^{(i, \mathbf{g}, 1)}=\left.\operatorname{rank}\left(\mathbf{M}_{\Delta}^{(i)} \mathbf{R}_{h}\right)\right|_{\mathbf{g}} \\
& =\left.\operatorname{rank}\left(\mathbf{M}_{\Delta}^{(i)} \mathbf{B}\right)\right|_{\mathbf{g}} \\
& =\sum_{t=1}^{T}\left(\sum_{k=1}^{K}\left(\left(L_{h}^{(k)}+1\right) \delta\left(\mathbf{g}^{(k, t)} \neq \mathbf{0}\right)\right)\right)
\end{aligned}
$$

2) For $N_{F}=\sum_{k=1}^{K}\left(\min \left(L_{h}^{(k)}+1, L_{g}^{(k)}+1\right)\right)$, we have (26)

Denote the PEP conditioned on channel $\left\{\mathbf{g}_{f}^{(i, k, t)}, k=1, \ldots, K, t=1, \ldots, T\right\}$ as $\alpha^{(i \mid \mathbf{g})}$, i.e.,

$$
\alpha^{(i \mid \mathbf{g})}=\operatorname{Pr}\left(\begin{array}{l|l}
\mathbf{x}^{(i)} \rightarrow \tilde{\mathbf{x}}^{(i)} & \begin{array}{l}
\left\{\mathbf{g}_{f}^{(i, k, t)}\right. \\
k=1, \ldots, K \\
t=1, \ldots, T
\end{array}
\end{array}\right) .
$$

$\left\{\mathbf{g}_{f}^{(i, k, t)}, k=1, \ldots, K, t=1, \ldots, T\right\}$, we need to calculate

$$
\alpha^{(i, b)}=\oiiint \alpha^{(i \mid \mathbf{g})} m_{\mathbf{g}} d \mathbf{g}
$$

where $d \mathbf{g}=\prod_{k=1}^{K} \prod_{t=1}^{T} \prod_{l_{g}=0}^{L_{g}^{(k)}} d g_{l_{g}}^{(k, t)}$, and $m_{\mathbf{g}}$ is the probability density function of $g$. (28) can be rewritten as the form of discrete summations

$$
\alpha^{(i, b)}=\sum \ldots \sum \alpha^{(i \mid \mathbf{g})} m_{\mathbf{g}} \mathbf{\Delta} \mathbf{g}
$$

where $\Delta \mathrm{g}$ is the discrete multiple integration unit area.

It is useful to derive the inequality (30), where $\left\{\lambda_{m}\right\}$ are non-zero eigenvalues of $\left(\mathbf{R}_{\mathbf{h}}\right)^{1 / 2} \mathrm{M}_{\Delta}^{(i)}\left(\mathbf{R}_{\mathbf{h}}\right)^{1 / 2}, N^{(i)}=$ $\operatorname{rank}\left(\left(\mathbf{R}_{\mathbf{h}}\right)^{1 / 2} \mathrm{M}_{\Delta}^{(i)}\left(\mathbf{R}_{\mathbf{h}}\right)^{1 / 2}\right)=\operatorname{rank}\left(\mathrm{M}_{\Delta}^{(i)} \mathbf{R}_{\mathbf{h}}\right), g^{(i, \Delta)}$ is defined in (31), $\rho_{\mathbf{g}}$ is symbol signal-to-noise ratio (SNR) conditioned on $\mathbf{g}$, and $\mu$ is some positive constant depending on the system structure.

Denote $\alpha^{(i \mid \mathbf{g})}$ in (27) for the case of $N_{F} \geq$ 


$$
\begin{aligned}
& N^{(i, \mathbf{g}, 2)}=\left.\operatorname{rank}\left(\mathbf{M}_{\Delta}^{(i)} \mathbf{R}_{h}\right)\right|_{\mathbf{g}}=\operatorname{rank}\left(\mathbf{M}_{\Delta}^{(i)} \mathbf{R}_{h}\right)=\sum_{t=1}^{T}\left(\sum_{k=1}^{K}\left(\min \left(L_{h}^{(k)}+1, L_{g}^{(k)}+1\right) \delta\left(\mathbf{g}^{(k, t)} \neq 0\right)\right)\right) \\
& \left(\operatorname{det}\left(\left(\mathbf{R}_{\Delta}^{(i)}\right)^{-1} \mathbf{R}_{\mathbf{h}}\right)\right)^{-1}=\left(\operatorname{det}\left(\left(\mathbf{R}_{\Delta}^{(i)}\right)^{-1} \mathbf{R}_{\mathbf{h}}\right)\right)^{-1} \\
& =\left(\operatorname{det}\left(g^{(i, \Delta)} P_{1}\left(\mathbf{R}_{\mathbf{h}}\right)^{1 / 2} \mathrm{M}_{\Delta}^{(i)}\left(\mathbf{R}_{\mathbf{h}}\right)^{1 / 2}+\mathbf{I}_{T \sum_{k=1}^{K}\left(L_{h}^{(k)}+1\right)}\right)\right)^{-1} \\
& =\prod_{m=1}^{N^{(i)}}\left(1+\mu \rho_{\mathbf{g}} \lambda_{m}\right)^{-1} \leq\left(\mu \min \left\{\lambda_{m}\right\} \rho_{\mathbf{g}}\right)^{-N^{(i)}} \text {. } \\
& g^{(i, \Delta)}=\left(2\left(\sum_{t=1}^{T}\left(N_{F}+\frac{1}{1+P_{1}} \sum_{k=1}^{K}\left(P_{2}^{(k)}\left(\mathbf{g}_{f}^{(i, k, t)}\right)^{\mathcal{H}} \mathbf{g}_{f}^{(i, k, t)}\right)\right)\right)\right)^{-1}
\end{aligned}
$$

$\sum_{k=1}^{K}\left(L_{g}^{(k)}+L_{h}^{(k)}+1\right)$ as $\alpha_{1}^{(i \mid \mathbf{g})}$. Denote $\alpha^{(i \mid \mathbf{g})}$ for the case of $N_{F}=\sum_{k=1}^{K}\left(\min \left(L_{h}^{(k)}+1, L_{g}^{(k)}+1\right)\right)$ as $\alpha_{2}^{(i \mid \mathbf{g})}$. Using (25), (26), and (30), (27) can be bounded as

$$
\alpha_{1}^{(i \mid \mathbf{g})} \leqslant d^{(i, \mathbf{g}, 1)}\left(\rho_{\mathbf{g}}\right)^{-N^{(i, \mathbf{g}, 1)}}=\mathbf{t}^{\left(i, \rho_{\mathbf{g}}, 1\right)}
$$

for $N_{F} \geq \sum_{k=1}^{K}\left(L_{g}^{(k)}+L_{h}^{(k)}+1\right)$ and

$$
\alpha_{2}^{(i \mid \mathbf{g})} \leqslant d^{(i, \mathbf{g}, 2)}(\rho)^{-N^{(i, \mathbf{g}, 2)}}=\mathbf{t}^{\left(i, \rho_{\mathbf{g}}, 2\right)},
$$

for $N_{F}=\sum_{k=1}^{K}\left(\min \left(L_{h}^{(k)}+1, L_{g}^{(k)}+1\right)\right)$, where $d^{(i, \mathbf{g}, 1)}$ and $d^{(i, \mathbf{g}, 2)}$ are two positive values conditioned on $\mathbf{g}$. In high SNR, $\left(\rho_{\mathbf{g}}\right)^{-N^{(i, \mathbf{g}, 1)}}$ and $\left(\rho_{\mathbf{g}}\right)^{-N^{(i, \mathbf{g}, 1)}}$ dominate $\mathbf{t}^{\left(i, \rho_{\mathbf{g}}, 1\right)}$ and $\mathbf{t}^{\left(i, \rho_{\mathbf{g}}, 2\right)}$ instead of $d^{(i, \mathbf{g}, 1)}$ and $d^{(i, \mathbf{g}, 2)}$, respectively. Using (25), (26), (32), and (33), we have the following observations: If $\mathbf{g} \neq \mathbf{0}$, we have $N^{(i, \mathbf{g}, 1)}>N^{(i, \mathbf{g}, 2)}$ and $\alpha_{1}^{(i \mid \mathbf{g})}<\alpha_{2}^{(i \mid \mathbf{g})}$, and the slope for $\log \left(\mathbf{t}^{\left(i, \rho_{\mathbf{g}}, 1\right)}\right)$ versus SNR conditioned on $\mathbf{g}$ is larger than that for $\log \left(\mathbf{t}^{\left(i, \rho_{\mathbf{g}}, 2\right)}\right)$ versus SNR conditioned on $\mathbf{g}$. If $\mathbf{g}=\mathbf{0}, N^{(i, \mathbf{g}, 1)}=N^{(i, \mathbf{g}, 2)}$, it means that communication links from relay nodes to the destination node are completely disconnected (which usually happens at very low probability), and DSTFC cannot provide any help in this case.

Using the above mentioned results, we conclude that the freedom order for average PEP (28) or (29) under $N_{F} \geq$ $\sum_{k=1}^{K}\left(L_{g}^{(k)}+L_{h}^{(k)}+1\right)$ is larger than that under $N_{F}=$ $\sum_{k=1}^{K}\left(\min \left(L_{h}^{(k)}+1, L_{g}^{(k)}+1\right)\right)$.

\section{Simulation Results and Discussions}

In this section, we present Monte-Carlo simulation results to demonstrate the error-rate performance of the proposed schemes. We assume that the underlying frequency-selective channels follow uniform power delay profile, and remain constant within a fixed integer number of OFDM blocks, denoted as the channel change interval $(C C I)$, and change independently from one block to another block. The following are further assumed in all simulations:

- $L_{h}^{(1)}=\ldots=L_{h}^{(K)}=L_{h}$ and $L_{g}^{(1)}=\ldots=L_{g}^{(K)}=L_{g}$.

- $P_{1}=P$ and $P_{2}^{(1)}=\ldots=P_{2}^{(K)}=\frac{P}{K}$.

- CCIs for source-to-relay and relay-to-destination channels are equal to 1.

- Before DSTFC precoding, data symbols are modulated using 4-QAM modulation.

The horizontal axes of performance figures is $10 \log _{10} P$. Note the average symbol SNR is proportional to $P$.

In Fig. 1 , for $T=1$ and $K=2$, we compare the bit error rate (BER) performance of our proposed DSFC with that proposed in $[6]^{1}$. From Figure 1, it is observed that,

- The performance of DSFC for the case of $L_{h}=L_{g}=2$ is worse than that for the cases of $\left(L_{h}=5\right)>\left(L_{g}=2\right)$ and $\left(L_{h}=2\right)<\left(L_{g}=5\right)$,

- The performance of DSFC for the case of $\left(L_{h}=5\right)>$ $\left(L_{g}=2\right)$ is better than that for the case of $\left(L_{h}=2\right)<$ $\left(L_{g}=5\right)$,

- The performance of DSFC for the case that $N_{F}$ is set to at least $K\left(L_{h}+L_{g}+1\right)$ is better than for the case that $N_{F}$ is set to at least $K \min \left\{L_{h}+1, L_{g}+1\right\}$.

The second observation implies that the extra frequencyselective order for source-to-relay paths provided stronger diversity advantages over that for relay-to-destination paths. This phenomenon incurs due to the existing relay noises, and thus we would provide the following conjecture:

Conjecture 1: Due to the deterioration incurred by relay noises, the degradation of the conditional diversity order of DSFC and DSTFC in frequency-selective fading relay channels is much larger than that of DSTC in frequency-flat fading relay channels. This effect may be more significant in low and medium SNR regions.

${ }^{1}$ We remark that the discussions in [6] are based on general discrete frequency-selective channel models (channel taps are not necessarily uniformly spaced), while our discussions are based on SSTDL as described in Section II. In the comparisons of Figure 1, the BER performance results for DSFC approaches of Zhang, et al. are also based on the same SSTDL frequency-selective channel models. 


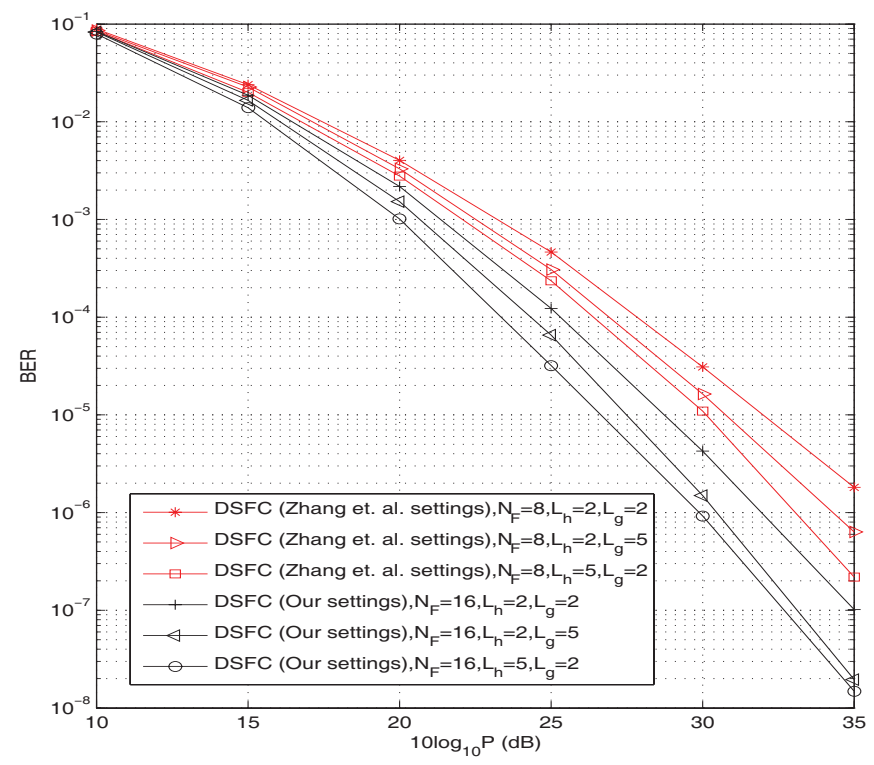

Fig. 1. Performance comparison of proposed DSFCs with those in [6] $\left(N_{C}=\right.$ $64)$.

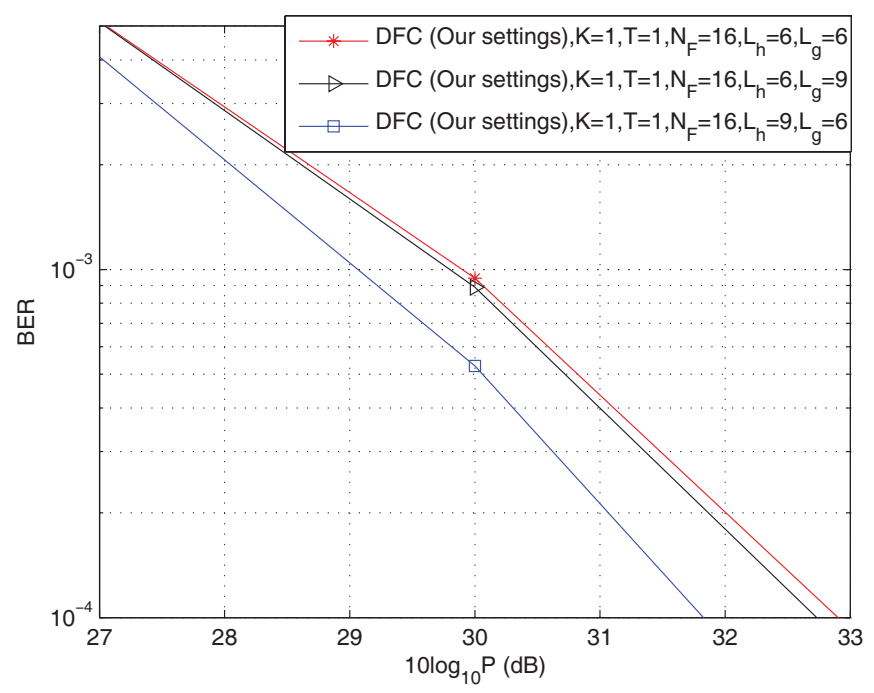

Fig. 2. BER Performance of DFCs $\left(N_{C}=32\right)$.

As mentioned in Section III, the authors of [6] have stated that the achievable diversity order based on average PEP is $K \min \left\{L_{h}+1, L_{g}+1\right\}$, and the corresponding minimum $N_{F}$ is set to be at least $K \min \left\{L_{h}+1, L_{g}+1\right\}$. However, according to our results based on the conditional PEP bound in Section III, we have stated that the achievable diversity order based one-side-channel conditional PEP is $K \max \left\{L_{h}+1, L_{g}+1\right\}$, and the corresponding minimum $N_{F}$ is set to be at least $K\left(L_{h}+L_{g}+1\right)$. Observations from Fig. 1 support our statements.

For $K=1$ and $T=1$, DSTFC becomes frequency-only coding, which can be termed as distributed frequency code (DFC). In Fig. 2, we investigate the BER performance of DFC under different frequency-selective channel conditions. It is observed that,

- The performance of DFC for the case of $L_{h}=L_{g}=6$

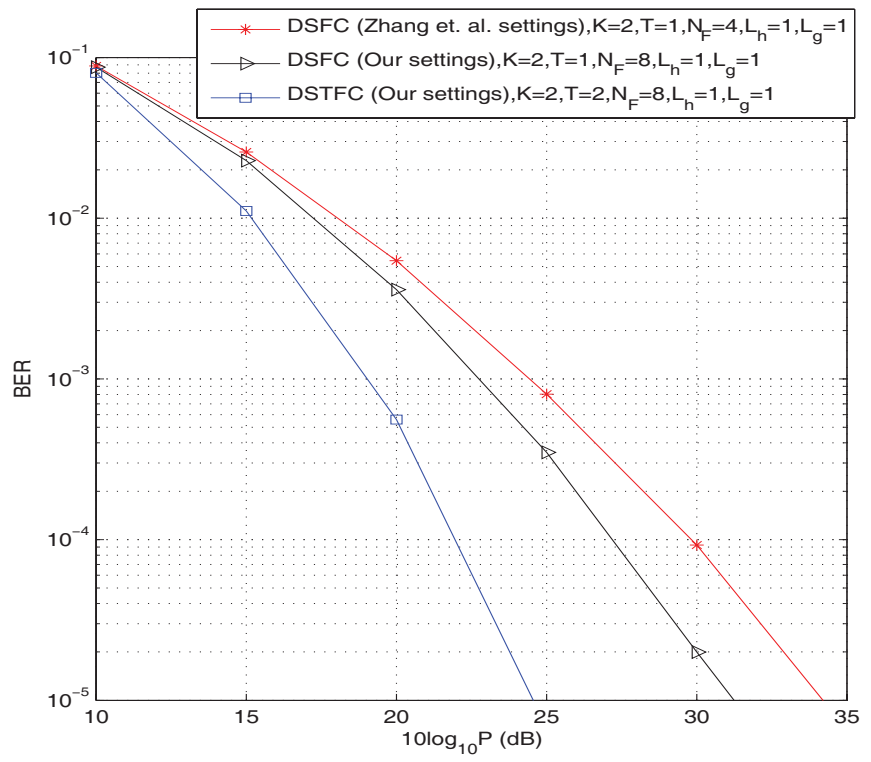

Fig. 3. Performance comparison of DSTFC and DSFCs $\left(N_{C}=32\right)$.

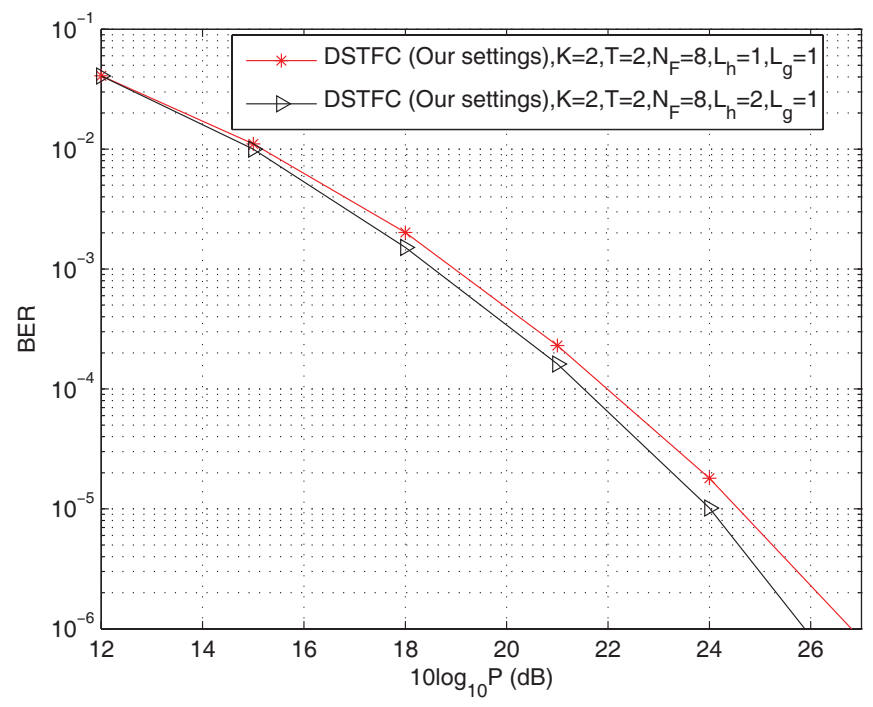

Fig. 4. Performance of DSTFC under different channel conditions $\left(N_{C}=\right.$ $32)$.

is worse than that for the cases of $\left(L_{h}=6\right)<\left(L_{g}=9\right)$ and $\left(L_{h}=9\right)>\left(L_{g}=6\right)$,

- The performance of DFC for the case of $\left(L_{h}=9\right)>$ $\left(L_{g}=6\right)$ is better than that for the case of $\left(L_{h}=6\right)<$ $\left(L_{g}=9\right)$,

The above results for single relay-case further confirm our diversity analysis in Section III.

Figure 3 illustrates performance comparisons between DSTFC and DSFC approaches. In time-varying channels (i.e, channel changes from one OFDM block to another), the proposed DSTFC demonstrates significant performance gains over DSFC at the price of longer decoding delay and higher computational complexity. In Fig. 4, the BER performances of DSTFC under different channel conditions are compared, which further confirms that, the channel order differences between source-to-relay and relay-to-destination may contribute 
to extra gains in diversity performance as discussed in Section III.

\section{CONCLUSION}

We have proposed high-rate distributed space-timefrequency coding for AF OFDM-based cooperative relay networks over frequency-selective fading channels. Using both conditional and average pairwise error probability, we have analyzed DSTFC diversity performance in block time-varying frequency-selective fading channels and pointed out the significant differences between diversity performance in frequencyselective channels and that in frequency-flat fading channels. Such observations help obtain better design criteria for DSTFCs. We have shown that high-rate DSTFC may significantly outperform high-rate DSFC due to efficient exploitation of time diversity over multiple OFDM blocks.

\section{APPENDIX A}

\section{DERIVATION OF $\mathbf{A}_{h}^{(i)}$}

Denote $\mathbf{f}^{(i)}=\underbrace{\left[\omega^{p_{1}^{(i)}-1}, \ldots, \omega^{p_{N_{F}}^{(i)}-1}\right]^{\mathcal{T}}}_{N_{F}}$.

The term $H_{p}^{(k, t)} G_{p}^{(k, t)}$ is derived in (34), where $\left[\mathbf{z}_{p}^{(k)}\right]_{l_{2}+\left(L_{g}^{(k)}+1\right) l_{1}+1,1}=\omega^{-\left(l_{1}+l_{2}\right)(p-1)}$, $\left[\mathbf{b}^{(k, t)}\right]_{l_{2}+\left(L_{g}^{(k)}+1\right) l_{1}+1,1}=h_{\left(l_{1}\right)}^{(k, t)} g_{\left(l_{2}\right)}^{(k, t)}, \quad l_{1}=$ $0, \ldots, L_{h}^{(k)}, l_{2}=0, \ldots, L_{g}^{(k)}, \mathbf{b}^{(k, t)}=\psi^{(k, t)} \mathbf{h}^{(k, t)}$, and $\psi^{(k, t)}=\operatorname{diag}\left(\mathbf{I}_{L_{h}^{(k)}} \otimes \mathbf{g}^{(k, t)}\right)$. Therefore, we have (35), where $\left[\Omega^{(i, k)}\right]_{:, l_{2}+\left(L_{g}^{(k)}+1\right) l_{1}+1}=\left[\mathbf{f}^{(i)}\right]^{\cdot\left(l_{1}+l_{2}\right)}$.

Note that

$$
\begin{aligned}
& \overline{\mathbf{h}}_{f}^{(i)}=\sum_{k=1}^{K}\left(\beta^{(k)} \sqrt{P_{2}^{(k)}} \overline{\mathbf{h}}_{f}^{(i, k)}\right) \\
& =\left[\Xi^{(i, 1)}, \ldots, \Xi^{(i, T)}\right] \\
& =\mathrm{M}^{(i)} \mathbf{b} .
\end{aligned}
$$

In (36), each element can be written as

$$
\begin{aligned}
& \Xi^{(i, t)}=\sum_{k=1}^{K}\left(\beta^{(k)} \sqrt{P_{2}^{(k)}}\left[\mathbf{a}^{(i, k, t)} \circ \mathbf{h}_{f}^{(i, k, t)} \circ \mathbf{g}_{f}^{(i, k, t)}\right]^{\mathcal{T}}\right) \\
& =\mathbf{M}^{(i, t)} \mathbf{b}^{(t)}
\end{aligned}
$$

where

$$
\begin{gathered}
\mathbf{h}^{(t)}=\left[\left[\mathbf{h}^{(1, t)}\right]^{\mathcal{T}}, \ldots,\left[\mathbf{h}^{(K, t)}\right]^{\mathcal{T}}\right]^{\mathcal{T}}, \\
\mathbf{h}=\left[\left[\mathbf{h}^{(1)}\right]^{\mathcal{T}}, \ldots,\left[\mathbf{h}^{(T)}\right]^{\mathcal{T}}\right]^{\mathcal{T}}, \\
\mathbf{M}^{(i)}=\operatorname{diag}\left(\mathrm{M}^{(i, 1)}, \ldots, \mathrm{M}^{(i, T)}\right), \\
\mathrm{M}^{(i, t)}=\left[\mathrm{M}^{(i, 1, t)}, \ldots, \mathrm{M}^{(i, K, t)}\right], \\
\mathrm{M}^{(i, k, t)}=\beta^{(k)} \sqrt{P_{2}^{(k)}} \operatorname{diag}\left(\mathbf{a}^{(i, k, t)}\right) \Omega^{(i, k)},
\end{gathered}
$$

$$
\begin{aligned}
\mathbf{b}^{(t)} & =\left[\left[\mathbf{b}^{(1, t)}\right]^{\mathcal{T}}, \ldots,\left[\mathbf{b}^{(K, t)}\right]^{\mathcal{T}}\right]^{\mathcal{T}}, \\
\mathbf{b} & =\left[\left[\mathbf{b}^{(1)}\right]^{\mathcal{T}}, \ldots,\left[\mathbf{b}^{(T)}\right]^{\mathcal{T}}\right]^{\mathcal{T}},
\end{aligned}
$$

$\mathbf{b}^{(t)}=\psi_{\mathbf{g}}^{(t)} \mathbf{h}^{(t)}, \psi_{\mathbf{g}}^{(t)}=\operatorname{diag}\left(\psi_{\mathbf{g}}^{(1, t)}, \ldots, \psi_{\mathbf{g}}^{(K, t)}\right), \mathbf{b}=\psi_{\mathbf{g}} \mathbf{h}$, and $\psi_{\mathbf{g}}=\operatorname{diag}\left(\psi_{\mathbf{g}}^{(1)}, \ldots, \psi_{\mathbf{g}}^{(T)}\right)$. Therefore, $\mathbf{A}_{h}^{(i)}$ is calculated as

$$
\begin{aligned}
& \mathbf{A}_{h}^{(i)}=\mathrm{M}^{(i)} \mathbf{b}(\mathbf{b})^{\mathcal{H}}\left(\mathrm{M}^{(i)}\right)^{\mathcal{H}} \\
& =\mathrm{M}^{(i)} \psi_{\mathbf{g}} \mathbf{h}(\mathbf{h})^{\mathcal{H}}\left(\psi_{\mathbf{g}}\right)^{\mathcal{H}}\left(\mathrm{M}^{(i)}\right)^{\mathcal{H}} .
\end{aligned}
$$

\section{APPENDIX B}

\section{Achievable Rank of $\mathrm{M}_{\Delta}^{(i)} \mathbf{R}_{\mathbf{h}}$}

In this section, we investigate the rank of $\mathrm{M}_{\Delta}^{(i)} \mathbf{R}_{\mathbf{h}}$. Under Assumption 1, we have $\mathbf{R}_{\mathbf{h}}=\operatorname{diag}\left(\mathbf{R}_{\mathbf{h}^{(1)}}, \ldots, \mathbf{R}_{\mathbf{h}^{(T)}}\right)$, where $\mathbf{R}_{\mathbf{h}^{(t)}}=E_{\mathbf{h}^{(t)}}\left(\mathbf{h}^{(t)}\left(\mathbf{h}^{(t)}\right)^{\mathcal{H}}\right)=\operatorname{diag}\left(\mathbf{R}_{\mathbf{h}^{(1, t)}}, \ldots, \mathbf{R}_{\mathbf{h}^{(K, t)}}\right)$, $\mathbf{R}_{\mathbf{h}^{(k, t)}}=E_{\mathbf{h}^{(k, t)}}\left(\mathbf{h}^{(k, t)}\left(\mathbf{h}^{(k, t)}\right)^{\mathcal{H}}\right)$. Further, denote $\Phi_{\mathbf{h}}=$ $\left(\mathbf{R}_{\mathbf{h}}\right)^{\frac{1}{2}}=\operatorname{diag}\left(\Phi_{\mathbf{h}^{(1)}}, \ldots, \Phi_{\mathbf{h}^{(T)}}\right)$, where $\Phi_{\mathbf{h}^{(t)}}=\left(\mathbf{R}_{\mathbf{h}^{(t)}}\right)^{\frac{1}{2}}=$ $\operatorname{diag}\left(\Phi_{\mathbf{h}^{(1, t)}}, \ldots, \Phi_{\mathbf{h}^{(K, t)}}\right), \Phi_{\mathbf{h}^{(k, t)}}=\left(\mathbf{R}_{\mathbf{h}^{(k, t)}}\right)^{\frac{1}{2}}, k=1, \ldots, K$, $t=1, \ldots, T$.

Recall that $\mathrm{M}_{\Delta}^{(i)}=\left(\psi_{\mathbf{g}}\right)^{\mathcal{H}}\left(\mathrm{M}^{(i)}\right)^{\mathcal{H}}\left(\Delta^{(i)}\right)^{\mathcal{H}} \Delta^{(i)} \mathrm{M}^{(i)} \psi_{\mathbf{g}}$, where

$$
\begin{gathered}
\psi_{\mathbf{g}}=\operatorname{diag}\left(\psi_{\mathbf{g}}^{(1)}, \ldots, \psi_{\mathbf{g}}^{(T)}\right), \\
\psi_{\mathbf{g}}^{(t)}=\operatorname{diag}\left(\psi_{\mathbf{g}}^{(1, t)}, \ldots, \psi_{\mathbf{g}}^{(K, t)}\right), \\
\psi_{\mathbf{g}}^{(k, t)}=\operatorname{diag}\left(\mathbf{I}_{L_{h}^{(k)}} \otimes \mathbf{g}^{(k, t)}\right) .
\end{gathered}
$$

Provided that $\Delta^{(i)}$ is to be full rank, i.e., $\operatorname{rank}\left(\Delta^{(i)}\right)=N_{F} T$, the following holds

$$
\begin{aligned}
& \operatorname{rank}\left(\mathrm{M}_{\Delta}^{(i)} \mathbf{R}_{\mathbf{h}}\right)=\operatorname{rank}\left(\Delta^{(i)} \mathrm{M}^{(i)} \psi_{\mathbf{g}} \Phi_{\mathbf{h}}\right) \\
& =\operatorname{rank}\left(\mathrm{M}^{(i)} \mathbf{B}\right)
\end{aligned}
$$

where $\mathbf{B}=\psi_{\mathbf{g}} \Phi_{\mathbf{h}}$.

\section{A. Investigation of $\mathbf{B}$}

Note that matrix $\mathbf{B}$ can be calculated as $\mathbf{B}=$ $\operatorname{diag}\left(\mathbf{B}^{(1)}, \ldots, \mathbf{B}^{(T)}\right)$, where $\mathbf{B}^{(t)}=\psi_{\mathbf{g}}^{(t)} \Phi_{\mathbf{h}^{(t)}}, \mathbf{B}^{(t)}=$ $\operatorname{diag}\left(\mathbf{B}^{(1, t)}, \ldots, \mathbf{B}^{(K, t)}\right), \mathbf{B}^{(k, t)}=\psi_{\mathbf{g}}^{(k, t)} \Phi_{\mathbf{h}^{(k, t)}}$. We know that $\mathbf{R}_{\mathbf{h}^{(k, t)}}, k=1, \ldots, K, t=1, \ldots, T$, are positive definite Hermitian matrices, thus all diagonal entries of $\mathbf{R}_{\mathbf{h}^{(k, t)}}$ are non-zero, so are $\Phi_{\mathbf{h}^{(1, t)}}, k=1, \ldots, K, t=1, \ldots, T$.

\section{B. Investigation of $\mathrm{M}^{(i)}$}

Recall that $\mathrm{M}^{(i)}=\operatorname{diag}\left(\mathrm{M}^{(i, 1)}, \ldots, \mathrm{M}^{(i, T)}\right)$, $\mathrm{M}^{(i, t)}=\left[\mathrm{M}^{(i, 1, t)}, \ldots, \mathrm{M}^{(i, K, t)}\right], \mathrm{M}^{(i, k, t)}=$ $\beta^{(k)} \sqrt{P_{2}^{(k)}} \operatorname{diag}\left(\mathbf{a}^{(i, k, t)}\right) \Omega^{(i, k)}$. We thus know that $\operatorname{rank}\left(\mathrm{M}^{(i)}\right)=\sum_{t=1}^{T} \operatorname{rank}\left(\mathrm{M}^{(i, t)}\right)$. To maximize the rank of $\mathrm{M}^{(i)}$, it is necessary to maximize the rank of each $\mathrm{M}^{(i, t)}$, respectively. This requires to properly design $\mathbf{a}^{(k, t)}$. Recall that $\left[\Omega^{(i, k)}\right]_{:, l_{2}+\left(L_{g}^{(k)}+1\right) l_{1}+1}=\left[\mathbf{f}^{(i)}\right]^{.\left(l_{1}+l_{2}\right)}$, where 


$$
\begin{gathered}
H_{p}^{(k, t)} G_{p}^{(k, t)}=\left(\sum_{l=o}^{L_{h}^{(k)}}\left(h_{(l)}^{(k, t)} e^{-j\left(2 \pi / N_{c}\right) l(p-1)}\right)\right)\left(\sum_{l=o}^{L_{g}^{(k)}}\left(g_{(l)}^{(k, t)} e^{-j\left(2 \pi / N_{c}\right) l(p-1)}\right)\right) \\
=\sum_{l_{1}=o}^{L_{h}^{(k)}}\left(\sum_{l_{2}=o}^{L_{g}^{(k)}}\left(h_{\left(l_{1}\right)}^{(k, t)} g_{\left(l_{2}\right)}^{(k, t)} e^{-j\left(2 \pi / N_{c}\right)(p-1)\left[l_{1}+l_{2}\right]}\right)\right)=\left[\mathbf{z}_{p}^{(k)}\right]^{\mathcal{T}} \mathbf{b}^{(k, t)} . \\
\mathbf{h}_{f}^{(i, k, t)} \circ \mathbf{g}_{f}^{(i, k, t)}=\left(\sum_{l_{1}=o}^{L_{h}^{(k)}}\left(h_{\left(l_{1}\right)}^{(k, t)}\left[\mathbf{f}^{(i)}\right]^{\left(l_{1}\right)}\right)\right)\left(\sum_{l_{2}=o}^{L_{g}^{(k)}}\left(g_{\left(l_{2}\right)}^{(k, t)}\left[\mathbf{f}^{(i)}\right]^{\left(l_{2}\right)}\right)\right)=\Omega^{(i, k)} \mathbf{b}^{(k, t)} .
\end{gathered}
$$

$\mathbf{f}^{(i)}=\underbrace{\left[\omega^{-\left(p_{1}^{(i)}-1\right)}, \ldots, \omega^{-\left(p_{N_{F}}^{(i)}-1\right)}\right]^{\mathcal{T}}}_{N_{F}}, l_{1}=0, \ldots, L_{h}^{(k)}$, and $l_{2}=0, \ldots, L_{g}^{(k)}$.

Assume that our design ensures $\left[\mathbf{f}^{(i)}\right]^{\cdot\left(a_{1}\right)}$ and $\left[\mathbf{f}^{(i)}\right]^{\cdot\left(a_{2}\right)}$ are linear-independent for all $a_{1} \neq a_{2}$, which can be achieved by choosing subcarrier indices $\left\{p_{a}^{(i)}\right\}$ such that $p_{a}^{(i)}+b=$ $p_{a+1}^{(i)}$, where $a=1, \ldots, N_{F}-1$. If $b=1$, adjacent subcarrier indices are chosen. Observing that $\left[\Omega^{(i, k)}\right]_{:, l_{2}+\left(L_{g}^{(k)}+1\right) l_{1}+1}=$ $\left[\mathbf{f}^{(i)}\right]^{\cdot\left(l_{1}+l_{2}\right)}$, where $l_{2}=0,1, \ldots, L_{g}^{(k)}, l_{1}=0,1, \ldots, L_{h}^{(k)}$, although $\Omega^{(i, k)}$ have $\left(L_{h}^{(k)}+1\right)\left(L_{g}^{(k)}+1\right)$ columns, there are only $\left(L_{g}^{(k)}+L_{h}^{(k)}+1\right)$ different columns regardless of different coefficients, since there are totally only $\left(L_{g}^{(k)}+L_{h}^{(k)}+1\right)$ different element-wise power $a$ for $\left[\mathbf{f}^{(i)}\right]^{\cdot(a)}$. This leads to $\operatorname{rank}\left(\Omega^{(i, k)}\right)=\left(L_{g}^{(k)}+L_{h}^{(k)}+1\right)$. Note that, in the case of $\operatorname{diag}\left(\mathbf{a}^{(k, t)}\right)=\mathbf{I}_{N_{F}}$, the maximal rank of $\mathrm{M}^{(i, t)}=\left[\beta^{(1)} \sqrt{P_{2}^{(1)}} \Omega^{(i, 1)}, \ldots, \beta^{(K)} \sqrt{P_{2}^{(K)}} \Omega^{(i, K)}\right]$ is $\max \left\{L_{g}^{(k)}+L_{h}^{(k)}+1, k=1, \ldots, K\right\}$. Now we set

$$
\mathbf{a}^{(i, k, t)}=\left[\mathbf{f}^{(i)}\right]^{\cdot\left((k-1) L_{M}\right)}
$$

where $L_{M}>\max \left\{L_{g}^{(k)}+L_{h}^{(k)}+1, k=1, \ldots, K\right\}$. Using $\mathbf{a}^{(i, k, t)}$ as specified in (40), $\mathrm{M}^{(i, t)}$ has $\sum_{k=1}^{K}\left(L_{g}^{(k)}+L_{h}^{(k)}+1\right)$ linear independent columns, in other words, $\operatorname{rank}\left(\mathrm{M}^{(i, t)}\right)=$ $\sum_{k=1}^{K}\left(L_{g}^{(k)}+L_{h}^{(k)}+1\right)$, for all $t=1, \ldots, T$.

\section{Rank of $\mathrm{M}^{(i)} \mathbf{B}$}

Now we are ready to investigate the rank of $\mathrm{M}^{(i)} \mathbf{B}$. We can write $\mathbf{M}^{(i)} \mathbf{B}$ as

$$
\mathbf{M}^{(i)} \mathbf{B}=\operatorname{diag}\left(\mathbf{M}_{\mathbf{B}}^{(i, 1)}, \ldots, \mathbf{M}_{\mathbf{B}}^{(i, T)}\right)
$$

where $\mathrm{M}_{\mathbf{B}}^{(i, t)}=\mathrm{M}^{(i, t)} \mathbf{B}^{(t)}=\left[\mathrm{M}_{\mathbf{B}}^{(i, 1, t)}, \ldots, \mathrm{M}_{\mathbf{B}}^{(i, K, t)}\right]$, and

$$
\begin{aligned}
& \mathbf{M}_{\mathbf{B}}^{(i, k, t)}=\mathbf{M}^{(i, k, t)} \mathbf{B}^{(k, t)} \\
& =\beta^{(k)} \sqrt{P_{2}^{(k)}} \operatorname{diag}\left(\mathbf{a}^{(i, k, t)}\right) \Omega^{(i, k)} \mathbf{B}^{(k, t)} \\
& =\beta^{(k)} \sqrt{P_{2}^{(k)}} \Omega_{\mathbf{a}}^{(i, k, t)} \mathbf{B}^{(k, t)} \\
& =\beta^{(k)} \sqrt{P_{2}^{(k)}} \Omega_{\mathbf{B}}^{(i, k, t)},
\end{aligned}
$$

where $\left[\Omega_{\mathbf{a}}^{(i, k, t)}\right]_{:, l_{2}+\left(L_{g}^{(k)}+1\right) l_{1}+1}=\left[\mathbf{f}^{(i)}\right]^{.\left(l_{1}+l_{2}+(k-1) L_{M}\right)}$, $\Omega_{\mathbf{B}}^{(i, k, t)}=\Omega_{\mathbf{a}}^{(i, k, t)} \mathbf{B}^{(k, t)}$.

Denote $\Omega_{\mathbf{a}\left(l_{1}\right)}^{(i, k, t)}=\left[\Omega_{\mathbf{a}}^{(i, k, t)}\right]_{:,\left(L_{g}^{(k)}+1\right) l_{1}+1: L_{g}^{(k)}+\left(L_{g}^{(k)}+1\right) l_{1}+1}$, and thus $\Omega_{\mathbf{a}}^{(i, k, t)}=\left[\begin{array}{c}\Omega_{\mathbf{a}(0)}^{(i, k, t)}, \ldots, \Omega_{\mathbf{a}\left(L_{h}^{(k)}\right)}^{(i, k, t)} \\ \left(L^{(k)}\right.\end{array}\right]$ Recall $\mathbf{B}^{(k, t)}=\psi_{\mathbf{g}}^{(k, t)} \Phi_{\mathbf{h}^{(1, t)}}$ and $\psi_{\mathbf{g}}^{(k, t)}=\operatorname{diag}\left(\mathbf{I}_{L_{h}^{(k)}} \otimes \mathbf{g}^{(k, t)}\right)$. We then obtain

$$
\Omega_{\mathbf{B}}^{(i, k, t)}=\left[\overline{\mathbf{g}}_{(0)}^{(i, k, t)}, \ldots, \overline{\mathbf{g}}_{\left(L_{h}^{(k)}\right)}^{(i, k, t)}\right] \Phi_{\mathbf{h}^{(k, t)}},
$$

where $\overline{\mathbf{g}}_{\left(l_{1}\right)}^{(i, k, t)}=\Omega_{\mathbf{a}\left(l_{1}\right)}^{(i, k, t)} \mathbf{g}^{(k, t)}, l_{1}=0, \ldots, L_{h}^{(k)}$.

$$
\begin{aligned}
& \overline{\mathbf{g}}_{\left(l_{1}\right)}^{(i, k, t)}=\Omega_{\mathbf{a}\left(l_{1}\right)}^{(i, k, t)} \mathbf{g}^{(k, t)} \\
& =\sum_{l_{2}=0}^{L_{g}^{(k)}}\left(g_{l_{2}}^{(k, t)}\left[\mathbf{f}^{(i)}\right]^{\left(l_{1}+l_{2}+(k-1) L_{M}\right)}\right) \\
& =\sum_{m_{2}=1}^{L_{g}^{N(k, t)}}\left(g_{\tau\left(m_{2}\right)}^{(k, t)}\left[\mathbf{f}^{(i)}\right]^{\left(l_{1}+\tau\left(m_{2}\right)+(k-1) L_{M}\right)}\right),
\end{aligned}
$$

where $L_{g}^{N(k, t)}$ is the total number of non-zero entries in vector $\mathbf{g}^{(k, t)}$, the non-zero entries of $\mathbf{g}^{(k, t)}$ are denoted as $g_{\tau(1)}^{(k, t)}, \ldots, g_{\tau\left(L_{g}^{N(k, t)}\right)}^{(k, t)}, 0 \leq \tau(1)<\tau(2)<\ldots<$ $\tau\left(L_{g}^{N(k, t)}\right) \leq L_{g}^{(k)}$. Based on Assumption 2, $L_{g}^{N(k, t)} \neq 0$ hold for all $1 \leq k \leq K$ and $1 \leq t \leq T$. Since $\overline{\mathbf{g}}_{\left(l_{1}\right)}^{(i, k, t)}$ is the linear combination of $L_{g}^{N(k, t)}$ linear independent columns $\left\{\left[\mathbf{f}^{(i)}\right]^{\left(l_{1}+\tau\left(m_{2}\right)+(k-1) L_{M}\right)} \mid 1 \leq m_{2} \leq L_{g}^{N(k, t)}\right\}, \overline{\mathbf{g}}_{\left(l_{1}\right)}^{(i, k, t)} \neq$ 0 holds for all $0 \leq l_{1} \leq L_{h}^{(k)}, 1 \leq k \leq K, 1 \leq t \leq T$, and $0 \leq l_{1} \leq L_{h}^{(k)}$. Note that $\left[\mathbf{f}^{(i)}\right]^{\cdot\left(l_{1}+\tau\left(L_{g}^{N(k, t)}\right)+(k-1) L_{M}\right)}$ does not exist in the linear combination within $\overline{\mathbf{g}}_{(0)}^{(i, k, t)}, \ldots, \mathbf{g}_{\left(l_{1}-1\right)}^{(i, k, t)}$, respectively, where $0 \leq l_{1} \leq L_{h}^{(k)}$. Thus, $\overline{\mathbf{g}}_{\left(l_{1}\right)}^{(i, k, t)}$ is linear independent to all $\mathbf{g}_{\left(\left(l_{2}\right)\right.}^{(i, k, t)}$ for $L_{h}^{(k)} \geq l_{1}>l_{2} \geq 0$. Using this 
recursive way, it can be proven that any two vectors within the set $\left\{\overline{\mathbf{g}}_{(0)}^{(i, k, t)}, \ldots, \overline{\mathbf{g}}_{\left(L_{h}^{(k)}\right)}^{(i, k, t)}\right\}$ are pairwise independent.

Now we can calculate the rank of $\Omega_{\mathbf{B}}^{(i, k, t)}$. Using $\operatorname{rank}\left(\Phi_{\mathbf{h}^{(k, t)}}\right)=L_{h}^{(k)}$, the following holds

$$
\begin{aligned}
& \operatorname{rank}\left(\Omega_{\mathbf{B}}^{(i, k, t)}\right) \\
& =\operatorname{rank}\left(\left[\overline{\mathbf{g}}_{(0)}^{(i, k, t)}, \ldots, \overline{\mathbf{g}}_{\left(L_{h}^{(k)}\right)}^{(i, k) t)}\right]\right)=L_{h}^{(k)}+1 .
\end{aligned}
$$

Further, note that the

Further, $\left.\begin{array}{c}\text { note } \\ {\left[\mathbf{f}^{(i)}\right]^{\cdot\left(l_{1}+\tau\left(m_{2}\right)+\left(k_{1}-1\right) L_{M}\right)},} \\ 1 \leq m_{2} \leq L_{g}^{N\left(k_{1}, t\right)}, 0 \leq l_{1} \leq L_{h}^{\left(k_{1}\right)}\end{array}\right\}$ and
the set $\left\{\begin{array}{l}{\left[\mathbf{f}^{(i)}\right]^{\cdot\left(l_{1}+\tau\left(m_{2}\right)+\left(k_{2}-1\right) L_{M}\right)}} \\ 1 \leq m_{2} \leq L_{g}^{N\left(k_{2}, t\right)}, 0 \leq l_{1} \leq L_{h}^{\left(k_{2}\right)}\end{array}\right\}$ do not have any common element vector, where $1 \leq k_{1} \neq k_{2} \leq K$, $L_{M}>\max \left\{\begin{array}{l}\left(L_{g}^{(k)}+L_{h}^{(k)}+1\right), \\ 1 \leq k \leq M\end{array}\right\}$, thus any element vector in $\left\{\mathbf{g}_{(0)}^{\left(i, k_{1}, t\right)}, \ldots, \overline{\mathbf{g}}_{\left(L_{h}^{(k)}\right)}^{\left(i, k_{1}, t\right)}\right\}$ is linear-independent to any element vector in $\left\{\mathbf{g}_{(0)}^{\left(i, k_{2}, t\right)}, \ldots, \overline{\mathbf{g}}_{\left(L_{h}^{(k)}\right)}^{\left(i, k_{2}, t\right)}\right\}$. Therefore,

$$
\operatorname{rank}\left(\mathrm{M}_{\mathbf{B}}^{(i, t)}\right)=\sum_{k=1}^{K} \operatorname{rank}\left(\Omega_{\mathbf{B}}^{(i, k, t)}\right)
$$

and

$$
\begin{aligned}
& \operatorname{rank}\left(\mathrm{M}_{\Delta}^{(i)} \mathbf{R}_{\mathbf{h}}\right)=\operatorname{rank}\left(\mathrm{M}^{(i)} \mathbf{B}\right) \\
& =\sum_{t=1}^{T} \operatorname{rank}\left(\mathrm{M}_{\mathbf{B}}^{(i, t)}\right) \\
& =T \sum_{k=1}^{K}\left(L_{h}^{(k)}+1\right)
\end{aligned}
$$

To achieve the achievable rank provided in (47), there are $\sum_{k=1}^{K}\left(L_{g}^{(k)}+L_{h}^{(k)}+1\right)$ available element vectors in set $\left\{\left[\mathbf{f}^{(i)}\right]^{\cdot\left(l_{1}+l_{2}+(k-1) L_{M}\right)}\right\}$, and note that those element vectors have to be linear-independent. Thus we have to choose

$$
N_{F} \geq \sum_{k=1}^{K}\left(L_{g}^{(k)}+L_{h}^{(k)}+1\right)
$$

\section{ACKNOWLEDGEMENTS}

The authors would like to thank the editor and anonymous reviewers for invaluable comments and suggestions.

\section{REFERENCES}

[1] T. Cover and A. El-Gamal, "Capacity theorems for the relay channel," IEEE Trans. Inf. Theory, vol. 25, no. 5, pp. 572-584, Sep. 1979.

[2] J. Laneman and G. Wornell, "Distributed space-time-coded protocols for exploiting cooperative diversity in wireless networks," IEEE Trans. Inf. Theory, vol. 49, no. 10, pp. 2415-2425, Oct. 2003.

[3] A. Sendonaris, E. Erkip, and B. Aazhang, "User cooperation diversitypart I: system description,” IEEE Trans. Commun., vol. 51, no. 21, pp. 1927-1938, Nov. 2003.

[4] P. A. Anghel and M. Kaveh, "On the performance of distributed spacetime coding systems with one and two non-regenerative relays," IEEE Trans. Wireless Commun., pp. 682-692, Mar. 2006.

[5] Y. Jing and B. Hassibi, "Diversity analysis of distributed spacetime codes in relay networks with multiple transmit/receive antennas,' EURASIP J. Advances in Signal Process., Jan. 2008.
[6] W. Zhang, Y. Li, X.-G. Xia, P. C. Ching, and K. B. Letaief, "Distributed space-frequency coding for cooperative diversity in broadband wireless ad hoc networks," IEEE Trans. Wireless Commun., vol. 7, no. 3, pp. 995-1003, Mar. 2008.

[7] K. G. Seddik and K. J. R. Liu, "Distributed space-frequency coding over broadband relay channels," IEEE Trans. Wireless Commun., vol. 7, no. 11, pp. 4748-4759, Nov. 2008.

[8] H. Wang, X.-G. Xia, and Q. Yin, "Distributed space-frequency codes for cooperative communication systems with multiple carrier frequency offsets," IEEE Trans. Wireless Commun., vol. 8, no. 2, pp. 1045-1055, Feb. 2009.

[9] W. Su, Z. Safar, and K. J. R. Liu, "Towards maximum achievable diversity in space, time, and frequency: performance analysis and code design," IEEE Trans. Wireless Commun., vol. 4, no. 4, pp. 1847-1857, July 2005

[10] O. Oguz, A. Zaidi, J. Louveaux, and L. Vandendorpe, "Distributed space-time-frequency block codes for multiple-access-channel with relaying," in Proc. IEEE GLOBECOM 2007, Nov. 2007, pp. 1729-1733.

[11] L. C. Tran, A. Mertins, and T. A. Wysocki, "Cooperative communication in space-time-frequency coded MB-OFDM UWB," in Proc. IEEE Vehicular Tech. Conf. 2008 Fall, Sep. 2008, pp. 1-5.

[12] W. Yang, Y. Cai, and L. Wang, "Distributed space-time-frequency coding for cooperative OFDM systems," Science in China Series F: Inf. Sciences, vol. 52, no. 12, pp. 2424-2432, Dec. 2009.

[13] S. Alamouti, "A simple transmitter diversity scheme for wireless communications," IEEE J. Sel. Areas Commun., pp. 1451-1458, Oct. 1998.

[14] V. Tarokh, H. Jafarkhani, and A. R. Calderbank, "Space-time block code from orthogonal designs," IEEE Trans. Inf. Theory, vol. 45, pp. 14561467, July 1999.

[15] Y. S. Choi, P. J. Voltz, and F. A. Cassara, "On channel estimation and detection for multicarrier signals in fast and selective Rayleigh fading channels," IEEE Trans. Commun., vol. 49, no. 8, pp. 1375-1387, Aug. 2001.

[16] X. Giraud, E. Boutillon, and J. C. Belfiore, "Algebraic tools to build modulation schemes for fading channels," IEEE Trans. Inf. Theory, vol. 43, pp. 938-952, May 1997.

[17] J. Boutros and E. Viterbo, "Signal space diversity: a power- and bandwidth-efficient diversity technique for the Rayleigh fading channel," IEEE Trans. Inf. Theory, vol. 44, pp. 1453-1467, July 1998.

[18] Y. Xin, Z. Wang, and G. B. Giannakis, "Space-time diversity systems based on linear constellation precoding," IEEE Trans. Wireless Commun., vol. 2, pp. 294-309, Mar. 2003.

[19] Z. Liu, Y. Xin, and G. B. Giannakis, "Linear constellation precoded OFDM with maximum multipath diversity and coding gains," IEEE Trans. Commun., vol. 51, no. 3, pp. 416-427, Mar. 2003.

[20] Y. Jing and B. Hassibi, "Distributed space-time coding in wireless relay networks," IEEE Trans. Wireless Commun., vol. 5, no. 12, pp. 3524 3536, Dec. 2006.

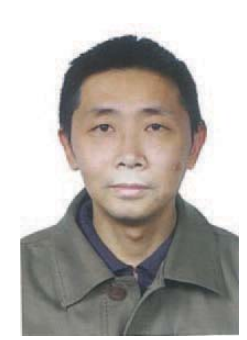

Jinsong Wu received the B.Eng. degree from Changsha Railway University (now part of Central South University), Changsha, China, in 1990, the M.A.Sc. degree from Dalhousie University, Halifax, NS, Canada, in 2001, the Ph.D. degree from Queen's University, Kingston, ON, Canada, in 2006, all in electrical engineering.

He experienced research and development positions of information technology or telecommunications industry with STONE in China, Great-Wall Computer in China, Nortel Networks in Canada, Philips Research North America in USA, and Sprint-Nextel in USA. He is currently working as a research fellow in University of Waterloo, Canada. His recent research interests lie in general communications theory and signal processing, space-time-frequency processing and coding, cooperative communications, iterative decoding or processing, communication optimization, and network protocols with applications. He has served as technical program committee members in IEEE Global Communications Conference 2008 and 2010 and IEEE International Conference on Communications 2009 and 2011. 


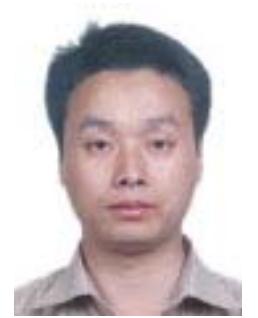

Honggang Hu received a B.S. degree in Mathematics in June 2000, and a B.E. degree in Electrical Engineering in June 2001 from the University of Science and Technology of China (USTC), Hefei, China, and the Ph.D. degree in Electrical Engineering from the Graduate School of Chinese Academy of Sciences, Beijing, China, in July 2005. From July 2005 to April 2007, he was a Postdoctoral Fellow at the Institute of Software, Chinese Academy of Sciences, Beijing, China. From August 2007 to July 2009, he was a Postdoctoral Fellow at the University of Waterloo, Canada. Since August 2009, he has been a Research Associate at the University of Waterloo, Canada. His research interests include pseudorandom sequences, cryptography, and coding theory.

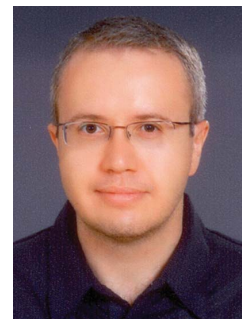

Murat Uysal was born in Istanbul, Turkey in 1973. $\mathrm{He}$ received the B.Sc. and the M.Sc. degree in electronics and communication engineering from Istanbul Technical University, Istanbul, Turkey, in 1995 and 1998, respectively, and the Ph.D. degree in electrical engineering from Texas A\&M University, College Station, Texas, in 2001. Since 2002, he has been with the Department of Electrical and Computer Engineering, University of Waterloo, Canada, where he is now an Associate Professor. He is currently on leave at Özyeğin University, Istanbul, Turkey. His general research interests lie in communications theory and signal processing for communications with special emphasis on wireless applications. Specific research areas include MIMO communication techniques, space-time coding, diversity techniques and coding for fading channels, cooperative communication, and free-space (wireless) optical communication. Dr. Uysal is an Associate Editor for IEEE TRANSACTIONS ON WIRELESS COMMUNICATIONS, IEEE COMMUNICATIONS LeTters, and Wireless Communications and Mobile Computing (WCMC) journal. In the past he served as a Guest Co-Editor for WCMC Special Issue on "MIMO Communications" (October 2004) and IEEE Journal on SElected AREAS In COMMUNiCATIONS Special Issue on "Optical Wireless Communications" (December 2009). Over the years, he has served on the technical program committee of more than 70 international conferences and workshops in the communications area. $\mathrm{He}$ recently co-chaired IEEE ICC 2007 Communication Theory Symposium and CCECE'08 Communications and Networking Symposium. He is currently serving as the Tutorial Chair of IEEE WCNC 2011. Dr. Uysal is a Senior IEEE member. 